\title{
A Dynamic Event-Triggered Approach to Recursive Non-fragile Filtering for Complex Networks with Sensor Saturations and Switching Topologies
}

\author{
Shaoying Wang, Zidong Wang, Hongli Dong and Yun Chen
}

\begin{abstract}
In this paper, the non-fragile filtering issue is addressed for complex networks (CNs) with switching topologies, sensor saturations and dynamic event-triggered communication protocol (DECP). Random variables obeying the Bernoulli distribution are utilized in characterizing the phenomena of switching topologies and stochastic gain variations. By introducing an auxiliary offset variable in the event-triggered condition, the DECP is adopted to reduce transmission frequency. The goal of this paper is to develop a non-fragile filter framework for considered CNs such that the upper bounds on the filtering error covariances are ensured. By virtue of mathematical induction, gain parameters are explicitly derived via minimizing such upper bounds. Moreover, a new method of analyzing the boundedness of given positive definite matrix is presented to overcome the challenges resulting from the coupled interconnected nodes, and sufficient conditions are established to guarantee the mean-square boundedness of filtering errors. Finally, simulations are given to prove the usefulness of our developed filtering algorithm.
\end{abstract}

Index Terms-Complex networks, non-fragile filter, dynamic event-triggered communication protocol, switching topologies, sensor saturations.

\section{INTRODUCTION}

Complex networks (CNs), comprised of large quantities of coupled nodes with a specific topology, have found applications in areas including social networks, biological networks, transportation network, communication network, and electrical power grids [14], [22], [27], [29], [36], [38], [40], [48], [50]. Among others, scale-free networks [43], random networks and small-world networks [44] have been well recognized as three representative categories of CNs. In general, it is quite difficult to fully access all the states of CNs owing to the coupling characteristic between nodes and the technological/physical

This work was supported in part by the National Natural Science Foundation of China under Grants 61703050, 61873058, 61873148, 61933007 and 61973102, the Natural Science Foundation of Heilongjiang Province of China under Grant ZD2019F001, and the Alexander von Humboldt Foundation of Germany. (Corresponding author: Hongli Dong)

S. Wang is with the College of Science, Binzhou University, Shandong 256603, China, and is also with the College of Electrical and Information Engineering, the Artificial Intelligence Energy Research Institute, Northeast Petroleum University, Daqing 163318, China (e-mail: shaoying2004@163. com)

Z. Wang is with the Department of Computer Science, Brunel University London, Uxbridge, Middlesex, UB8 3PH, United Kingdom. (e-mail: Zidong.Wangebrunel.ac.uk)

H. Dong is with the Artificial Intelligence Energy Research Institute, Northeast Petroleum University, Daqing 163318, China (e-mail: shiningdhl@gmail.com)

Y. Chen is with the Key Lab for IoT and Information Fusion Technology of Zhejiang, School of Automation, Hangzhou Dianzi University, Hangzhou 310018, China. (e-mail: yunchen@hdu. edu.cn) restrictions, and an alternative approach is therefore to infer network states according to partially available measurements.

Recently, the filtering issue of $\mathrm{CNs}$ has become a mainstream topic in the area of complexity science, see [11], [15], [30], [39], [46], [51]. In [15], the recursive filtering issue has been addressed for coupled stochastic CNs subject to the random disturbances and packet dropouts. In [50], the estimation problem has been investigated for time-delayed CNs with filter stability discussed.

In practice, phenomena of stochastic gain variations ( $\mathrm{SGVs}$ ) are inevitable in the process of filter realization due mainly to unexpected implementation errors such as rounding errors, analog-digital conversion errors, and programming errors [21]. The impact from SGVs, if not well handled, could lead to serious degradations on the overall filtering performance. As such, the last years have spotted a great surge of research interest on non-fragile filtering problems and some elegant results have appeared, see e.g. [23], [26], [28], [31], [37]. To mention a few, the non-fragile $H_{\infty}$ filter [21] and the distributed resilient recursive filter [26] have been designed for time-varying nonlinear systems.

Another frequently encountered phenomenon in engineering practice is the so-called sensor saturation that is primarily caused by the inherent physical constraints [49]. Correspondingly, a large amount of work has been acquired on sensorsaturation-resistant filtering problems, see [7], [18], [47], [54]. For example, in [47], the filtering and intermittent fault detection issues have been addressed for uncertain stochastic systems subject to sensor saturations. Nevertheless, to our best knowledge, non-fragile filtering issues for CNs with SGVs and sensor saturations have not been comprehensively considered.

In reality, it is quite common that the connection topology of CNs varies with time because of the changes of nodes in the spatially distributed networks [6], [11] and, accordingly, the filtering problem with switching topologies has attracted considerable attention from many researchers. Generally speaking, there have been mainly two approaches available in the literature to characterizing the switching behavior between coupling nodes, namely, the Bernoulli random variable approach [20] and the Markov chain approach [13]. In [6], a novel $H_{\infty}$ approach has been developed for nonlinear $\mathrm{CNs}$ subject to stochastic inner coupling, randomly varying topologies and measurement quantization, where topology changes of the $\mathrm{CNs}$ have been depicted by a Markov chain. Furthermore, an eventtriggered approach has been proposed in [13] for nonlinear CNs with missing measurements and randomly switching topologies. Unfortunately, for non-fragile filtering issues of 
CNs under switching topologies, the corresponding research has received little attention, which constitutes one motivation of our current investigation.

A practical issue for networked systems is how to improve the utilization efficiency of the inherently limited bandwidth for communication in a resource-scarce environment [1], [2], [25], [32], [34], [41], [42], [45], [52]. Among various transmission schemes that have been put forward to save network bandwidth, the so-called event-triggered strategy has received much research attention with the hope of averting unnecessarily frequent network transmissions while guaranteeing certain estimation performance. The basic concept of the so-called event-triggered transmission is that the data communication is executed only when the deviation between the current and the latest transmitted data meets a predefined condition [4], [5], [9], [13], [24], [33].

Very recently, the dynamic event-triggered communication protocol (DECP) has been put forward via introducing auxiliary variables [9], [10], [16], [53], and many important results have been given regarding filtering problems for various $\mathrm{CNs}$ under DECP, see e.g. [18], [19]. For instance, in [33], an eventtriggered filter has been designed for stochastic nonlinear systems by simultaneously taking into account linearization errors, packet losses, and time delays. In [9], a distributed set-membership filter has been devised in case of unknownbut-bounded process and measurement noises. Nevertheless, it is worth mentioning that little effort has been put so far into dynamic event-triggered filtering issues for $\mathrm{CNs}$, let alone the case where the SGVs, sensor saturations and switching topologies are also involved.

Based on aforementioned considerations, in this paper, we aim at investigating non-fragile filtering problems for timevarying $\mathrm{CNs}$ under DECP subject to sensor saturations and switching topologies. Some Bernoulli distributed random variables are used to describe phenomena of switching topologies and SGVs. The DECP is employed to schedule the data transmissions in a dynamical way. The main challenges we face lie in the following three aspects: 1) how to better model the phenomenon of DECP? 2) how to handle the impacts from sensor saturations, DECP and switching topologies on the design of recursive filter? and 3) how to analyze the meansquare boundedness of the filtering error? To overcome the listed challenges, we are dedicated to the development of a dynamic event-triggered non-fragile recursive filter such that upper bounds on the resulting filtering error covariances are obtained and then gain matrices are designed via minimizing such upper bounds.

The primary contributions we deliver in this paper are given as follows: 1) a dynamic event-triggered non-fragile filter, which is suitable for online implementation, is first proposed in the concurrence of sensor saturations, switching topologies and SGVs;2) sufficient conditions are provided to ensure mean-square boundedness of resulting filtering errors; and 3) a new technique, which analyzes the boundedness of a given positive definite matrix, is presented to tackle difficulties caused by the coupling of interconnected nodes.

Notation. $\|\cdot\|$ is the Euclidean norm or the spectral norm of vector or matrix ".". $P>0$ denotes that $P$ is a positive-definite matrix. $\operatorname{col}\{\cdot\}$ means to aggregate all the column vectors into a column vector. $A^{T}$ means the transpose of matrix $A . \operatorname{tr}(\mathrm{A})$ denotes the trace of matrix $A . \operatorname{sym}\{*\}$ represents $*+*^{T} . \mathbb{N}$ is the natural number set. $\mathbb{E}\{x\}$ represents the expectation of the random variable $x$. All matrices used in this paper are supposed to have appropriate dimensions.

\section{Problem Statement}

Consider a $\mathrm{CN}$ with switching topologies and sensor saturations:

$$
\left\{\begin{array}{c}
x_{k+1}^{(i)}=A_{k}^{(i)} x_{k}^{(i)}+c \sum_{j=1}^{L} \zeta_{k}^{(i j)} \Gamma x_{k}^{(j)}+B_{k}^{(i)} w_{k}^{(i)} \\
z_{k}^{(i)}=\varphi\left(C_{k}^{(i)} x_{k}^{(i)}\right)+v_{k}^{(i)} \quad i=1,2, \ldots, L
\end{array}\right.
$$

where $x_{k}^{(i)} \in \mathbb{R}^{n}$ is the system state, $z_{k}^{(i)} \in \mathbb{R}^{m}$ is the measurement output, $\Gamma$ represents an inner-coupling matrix, and $c>0$ refers to the overall coupling strength. $\zeta_{k}^{(i j)}$ are Bernoulli variables adopted to regulate the connection between nodes $i$ an $j$. Specifically, $\zeta_{k}^{(i j)}=1$ if node $i$ is connected with node $j$, and otherwise $\zeta_{k}^{(i j)}=0$. The probability distribution of $\zeta_{k}^{(i j)}$ satisfies $\mathbb{P}\left\{\zeta_{k}^{(i j)}=1\right\}=\bar{\zeta}_{k}^{(i j)}$ and $\mathbb{P}\left\{\zeta_{k}^{(i j)}=0\right\}=1-\bar{\zeta}_{k}^{(i j)} \cdot w_{k}^{(i)} \in \mathbb{R}^{w}$ and $v_{k}^{(i)} \in \mathbb{R}^{m}$ are zero-mean noises with covariances $Q_{k}^{(i)}$ and $R_{k}^{(i)}$, respectively. Matrices $A_{k}^{(i)}, B_{k}^{(i)}$ and $C_{k}^{(i)}$ are known.

The saturation function $\varphi: \mathbb{R}^{m} \rightarrow \mathbb{R}^{m}$ is defined as follows:

$$
\varphi(z)=\operatorname{col}\left\{\varphi_{1}\left(z_{1}\right), \varphi_{2}\left(z_{2}\right), \ldots, \varphi_{m}\left(z_{m}\right)\right\}
$$

where $\varphi_{i}\left(z_{i}\right)=\operatorname{sign}\left(z_{i}\right) \min \left\{\rho_{i},\left|z_{i}\right|\right\}, \operatorname{sign}(\cdot)$ is the signum function and $\rho_{i}(i=1,2, \cdots, m)$ represents the saturation level.

To reduce communication frequency, the DECP is adopted with its event generator function given by

$$
f\left(\mu_{k}^{(i)}, \sigma_{i}, \theta_{i}, \chi_{k}^{(i)}\right)=\left\|\mu_{k}^{(i)}\right\|-\sigma_{i}-\frac{\chi_{k}^{(i)}}{\theta_{i}}
$$

where $\mu_{k}^{(i)} \triangleq z_{k}^{(i)}-z_{k_{t}}^{(i)}, z_{k_{t}}^{(i)}$ is the latest transmitted measurement, and $\chi_{k}^{(i)}$ is an internal dynamical variable satisfying

$$
\chi_{k+1}^{(i)}=h_{i} \chi_{k}^{(i)}+\sigma_{i}-\left\|\mu_{k}^{(i)}\right\|
$$

where $0 \leq \sigma_{i}<1,0<h_{i}<1$ and $\theta_{i} \geq 1 / h_{i}$ are predefined scalars, and $\chi_{0}^{(i)} \geq 0$ is the initial value.

Since $z_{k}^{(i)}$ is sent to the remote filter only when $f\left(\mu_{k}^{(i)}, \sigma_{i}, \theta_{i}, \chi_{k}^{(i)}\right) \geq 0$, the triggering instant sequence $0 \leq$ $k_{0} \leq k_{1} \leq \cdots \leq k_{t} \leq \cdots$ is

$$
k_{t+1}=\inf _{k \in \mathbb{N}}\left\{k>k_{t} \mid f\left(\mu_{k}^{(i)}, \sigma_{i}, \theta_{i}, \chi_{k}^{(i)}\right) \geq 0\right\} .
$$

Accordingly, the available measurement at the filter, denoted by $\tilde{z}_{k}^{(i)}$, is described as

$$
\tilde{z}_{k}^{(i)}=z_{k_{t}}^{(i)}, k \in\left\{k_{t}, k_{t}+1, \ldots, k_{t+1}-1\right\} .
$$

Remark 1: Note that an auxiliary offset variable $\chi_{k}^{(i)}$, which evolves according to (4), has been exploited in the event triggering condition to dynamically adjust the inter-event time. 
As stated in [9], $\chi_{k}^{(i)}$ is a non-negative scalar when the parameters satisfy $\chi_{0}^{(i)} \geq 0,0<h_{i}<1$ and $\theta_{i} h_{i} \geq 1$. With this property, it is easy to see that the triggering times under DECP will be reduced when compared to those from the static event-triggered protocol, thereby further alleviating the network burden. It should also be pointed out that the DECP would reduce to the static protocol by setting $\theta_{i} \rightarrow \infty$.

Based on the available measurement $\tilde{z}_{k}^{(i)}$, the filter for node $i(i=1,2, \ldots, L)$ is constructed as follows:

$$
\left\{\begin{array}{l}
\hat{x}_{k+1 \mid k}^{(i)}=A_{k}^{(i)} \hat{x}_{k}^{(i)}+c \sum_{j=1}^{L} \bar{\zeta}_{k}^{(i j)} \Gamma \hat{x}_{k}^{(j)}, \\
\hat{x}_{k+1}^{(i)}=\hat{x}_{k+1 \mid k}^{(i)}+\tilde{G}_{k+1}^{(i)}\left(\tilde{z}_{k+1}^{(i)}-C_{k+1}^{(i)} \hat{x}_{k+1 \mid k}^{(i)}\right)
\end{array}\right.
$$

where $\hat{x}_{k+1 \mid k}^{(i)} \in \mathbb{R}^{n}$ and $\hat{x}_{k+1}^{(i)} \in \mathbb{R}^{n}$ denote the prediction and estimate of $x_{k+1}^{(i)}$, respectively. Note that $\tilde{G}_{k+1}^{(i)} \triangleq$ $G_{k+1}^{(i)}+\xi_{k+1}^{(i)} G_{s, k+1}^{(i)}$, where $G_{k+1}^{(i)}$ denotes the filter gain tobe-designed, $\xi_{k+1}^{(i)} G_{s, k+1}^{(i)}$ represents the stochastic uncertainty associated with the filter gain, $G_{s, k+1}^{(i)}$ is a determined matrix, and $\xi_{k+1}^{(i)} \in \mathbb{R}$ is the multiplicative noise with mean 0 and variance $\beta_{k+1}^{(i)}$.

Remark 2: It is observed from the proposed filter structure (7) that all the information about coupled configuration of the network, switching topologies, DECP and SGVs has been fully exploited. The stochastic parameter uncertainties $\xi_{k+1}^{(i)} G_{s, k+1}^{(i)}$ are utilized to characterize the resilience of the filter gain variations, which are inevitable in the process of filter realization owing to unexpected implementation errors. Note that the nonfragile filter proposed in this paper is quite different from that in [18], [26], where the distinctive difference lies in that 1) the main contribution of [18] is the design of a non-fragile $H_{\infty}$ filter for nonlinear CNs with gain perturbation satisfying $\Delta K_{i}=M_{i} F_{i, k} N_{i}$ and $\left.F_{i, k}^{T} F_{i, k} \leq I, 2\right)$ a distributed filter is proposed in [26] for a class of discrete time-varying systems with stochastic nonlinearities and sensor degradation with zero mean and bounded second-moment uncertainties $\triangle_{i j}(k)$, and 3 ) the objective here is to propose a dynamic event-triggered non-fragile recursive minimum-variance filtering algorithm for linear CNs with sensor saturations, switching topologies and SGVs.

For node $i$, the one-step prediction and filtering errors are, respectively, defined as

$$
\varepsilon_{k+1 \mid k}^{(i)}=x_{k+1}^{(i)}-\hat{x}_{k+1 \mid k}^{(i)}, \quad \varepsilon_{k+1}^{(i)}=x_{k+1}^{(i)}-\hat{x}_{k+1}^{(i)}
$$

and their corresponding error covariances are, respectively, denoted as

$$
\Xi_{k+1 \mid k}^{(i)}=\mathbb{E}\left[\varepsilon_{k+1 \mid k}^{(i)}\left(\varepsilon_{k+1 \mid k}^{(i)}\right)^{T}\right], \quad \Xi_{k+1}^{(i)}=\mathbb{E}\left[\varepsilon_{k+1}^{(i)}\left(\varepsilon_{k+1}^{(i)}\right)^{T}\right] .
$$

In this paper, we aim at designing filter (7) for CNs (1) such that, in case of sensor saturations, switching topologies, DECP and SGVs, upper bounds on $\Xi_{k+1}^{(i)}$ exist, i.e., $\Xi_{k+1}^{(i)} \leq \bar{\Xi}_{k+1}^{(i)}$, and $\bar{\Xi}_{k+1}^{(i)}$ is minimized through properly choosing parameter $G_{k+1}^{(i)}$. Moreover, sufficient conditions for mean-square boundedness of estimation errors are established.

\section{Main Results}

\section{A. Design of Dynamic Event-Triggered Non-fragile Filter}

In the first place, some useful lemmas are given for subsequent analysis.

Lemma 1: $O_{k}^{(i)} \triangleq \mathbb{E}\left[x_{k}^{(i)}\left(x_{k}^{(i)}\right)^{T}\right]$ has the following recursion:

$$
\begin{aligned}
O_{k+1}^{(i)}= & A_{k}^{(i)} O_{k}^{(i)}\left(A_{k}^{(i)}\right)^{T}+c^{2} \Phi_{11, k} \\
& +\Phi_{12, k}+\Phi_{12, k}^{T}+B_{k}^{(i)} Q_{k}^{(i)}\left(B_{k}^{(i)}\right)^{T}
\end{aligned}
$$

and, for a given positive scalar $\pi_{1}$, its upper bound can be calculated as

$$
\begin{aligned}
\bar{O}_{k+1}^{(i)}= & \left(1+\pi_{1}\right) A_{k}^{(i)} \bar{O}_{k}^{(i)}\left(A_{k}^{(i)}\right)^{T}+B_{k}^{(i)} Q_{k}^{(i)}\left(B_{k}^{(i)}\right)^{T} \\
& +\left(1+\pi_{1}^{-1}\right) c^{2} \sum_{j=1}^{L}\left(M_{i} \bar{\zeta}_{k}^{(i j)}+S_{k}^{(i j)}\right) \Gamma \bar{O}_{k}^{(j)} \Gamma^{T}
\end{aligned}
$$

where

$$
\begin{aligned}
& \Phi_{11, k} \triangleq \mathbb{E}\left[\left(\sum_{j=1}^{L} \zeta_{k}^{(i j)} \Gamma x_{k}^{(j)}\right)\left(\sum_{j=1}^{L} \zeta_{k}^{(i j)} \Gamma x_{k}^{(j)}\right)^{T}\right], \\
& \Phi_{12, k} \triangleq \mathbb{E}\left[A_{k}^{(i)} x_{k}^{(i)}\left(c \sum_{j=1}^{L} \zeta_{k}^{(i j)} \Gamma x_{k}^{(j)}\right)^{T}\right], \\
& S_{k}^{(i j)} \triangleq \bar{\zeta}_{k}^{(i j)}\left(1-\bar{\zeta}_{k}^{(i j)}\right), M_{i} \triangleq \sum_{j=1}^{L} \bar{\zeta}_{k}^{(i j)} .
\end{aligned}
$$

Proof: Based on (1) and the definition of $O_{k}^{(i)}$, it is quite straightforward to obtain (8).

Next, expanding the term $\Phi_{11, k}$, we have

$$
\begin{aligned}
\Phi_{11, k}= & \bar{\zeta}_{k}^{(i 1)} \Gamma O_{k}^{(1)} \Gamma^{T}+\cdots+\bar{\zeta}_{k}^{(i 1)} \bar{\zeta}_{k}^{(i L)} \Gamma \mathbb{E}\left[x_{k}^{(1)}\left(x_{k}^{(L)}\right)^{T}\right] \Gamma^{T} \\
& +\cdots+\bar{\zeta}_{k}^{(i L)} \bar{\zeta}_{k}^{(i 1)} \Gamma \mathbb{E}\left[x_{k}^{(L)}\left(x_{k}^{(1)}\right)^{T}\right] \Gamma^{T}+\cdots \\
& +\bar{\zeta}_{k}^{(i L)} \Gamma O_{k}^{(L)} \Gamma^{T} \\
= & \sum_{j=1}^{L} S_{k}^{(i j)} \Gamma O_{k}^{(j)} \Gamma^{T}+\sum_{j=1}^{L} \sum_{m=1}^{L} \bar{\zeta}_{k}^{(i j)} \bar{\zeta}_{k}^{(i m)} \\
& \times \Gamma \mathbb{E}\left[x_{k}^{(j)}\left(x_{k}^{(m)}\right)^{T}\right] \Gamma^{T}
\end{aligned}
$$

From the following elementary inequality

$$
a b^{T}+b a^{T} \leq \pi a a^{T}+\pi^{-1} b b^{T}(\pi>0),
$$

we have

$$
\begin{aligned}
& \sum_{j=1}^{L} \sum_{m=1}^{L} \bar{\zeta}_{k}^{(i j)} \bar{\zeta}_{k}^{(i m)} \Gamma \mathbb{E}\left[x_{k}^{(j)}\left(x_{k}^{(m)}\right)^{T}\right] \Gamma^{T} \\
= & \frac{1}{2} \sum_{j=1}^{L} \sum_{m=1}^{L} \bar{\zeta}_{k}^{(i j)} \bar{\zeta}_{k}^{(i m)} \Gamma \mathbb{E}\left[x_{k}^{(j)}\left(x_{k}^{(m)}\right)^{T}+x_{k}^{(m)}\left(x_{k}^{(j)}\right)^{T}\right] \Gamma^{T} \\
\leq & \frac{1}{2} \sum_{j=1}^{L} \sum_{m=1}^{L} \bar{\zeta}_{k}^{(i j)} \bar{\zeta}_{k}^{(i m)} \Gamma \mathbb{E}\left[x_{k}^{(j)}\left(x_{k}^{(j)}\right)^{T}+x_{k}^{(m)}\left(x_{k}^{(m)}\right)^{T}\right] \Gamma^{T} \\
= & \frac{1}{2} \sum_{m=1}^{L} \bar{\zeta}_{k}^{(i m)} \sum_{j=1}^{L} \bar{\zeta}_{k}^{(i j)} \Gamma \mathbb{E}\left[x_{k}^{(j)}\left(x_{k}^{(j)}\right)^{T}\right] \Gamma^{T}
\end{aligned}
$$




$$
\begin{aligned}
& +\frac{1}{2} \sum_{j=1}^{L} \bar{\zeta}_{k}^{(i j)} \sum_{m=1}^{L} \bar{\zeta}_{k}^{(i m)} \Gamma \mathbb{E}\left[x_{k}^{(m)}\left(x_{k}^{(m)}\right)^{T}\right] \Gamma^{T} \\
= & M_{i} \sum_{j=1}^{L} \bar{\zeta}_{k}^{(i j)} \Gamma O_{k}^{(j)} \Gamma^{T} .
\end{aligned}
$$

Similarly, the term $\Phi_{12, k}+\Phi_{12, k}^{T}$ is rewritten as

$$
\begin{aligned}
& \Phi_{12, k}+\Phi_{12, k}^{T} \\
\leq & \pi_{1} \mathbb{E}\left[A_{k}^{(i)} x_{k}^{(i)}\left(x_{k}^{(i)}\right)^{T}\left(A_{k}^{(i)}\right)^{T}\right] \\
& +\pi_{1}^{-1} \mathbb{E}\left[\left(c \sum_{j=1}^{L} \zeta_{k}^{(i j)} \Gamma x_{k}^{(j)}\right)\left(c \sum_{j=1}^{L} \zeta_{k}^{(i j)} \Gamma x_{k}^{(j)}\right)^{T}\right] \\
= & \pi_{1} A_{k}^{(i)} O_{k}^{(i)}\left(A_{k}^{(i)}\right)^{T}+\pi_{1}^{-1} c^{2} \Phi_{11, k} .
\end{aligned}
$$

Then, it follows from (10), (12) and (13) that

$$
\begin{aligned}
O_{k+1}^{(i)} \leq & \left(1+\pi_{1}\right) A_{k}^{(i)} O_{k}^{(i)}\left(A_{k}^{(i)}\right)^{T}+B_{k}^{(i)} Q_{k}^{(i)}\left(B_{k}^{(i)}\right)^{T} \\
& +\left(1+\pi_{1}^{-1}\right) c^{2} \sum_{j=1}^{L}\left(M_{i} \bar{\zeta}_{k}^{(i j)}+S_{k}^{(i j)}\right) \Gamma O_{k}^{(j)} \Gamma^{T}
\end{aligned}
$$

which implies that (9) holds.

Remark 3: In Lemma 1, the expression of the state covariance is obtained as shown in (8). Unfortunately, it is literally difficult to calculate the exact value via (8) owing to the existence of the unknown terms $\Phi_{12, k}+\Phi_{12, k}^{T}$. To deal with such an issue, an upper bound on the state covariance is given in (9) by using (11).

For node $i$, let us first focus our attention on the exact values of the one-step prediction and filtering error covariances $\Xi_{k+1 \mid k}^{(i)}$ and $\Xi_{k+1}^{(i)}$.

Lemma 2: The recursion of $\Xi_{k+1 \mid k}^{(i)}$ is

$$
\begin{aligned}
\Xi_{k+1 \mid k}^{(i)}= & A_{k}^{(i)} \Xi_{k}^{(i)}\left(A_{k}^{(i)}\right)^{T}+c^{2} \sum_{j=1}^{L} S_{k}^{(i j)} \Gamma O_{k}^{(j)} \Gamma^{T}+\mathcal{N}_{11, k}^{(i)} \\
& +\mathcal{N}_{12, k}^{(i)}+\left(\mathcal{N}_{12, k}^{(i)}\right)^{T}+B_{k}^{(i)} Q_{k}^{(i)}\left(B_{k}^{(i)}\right)^{T}
\end{aligned}
$$

where

$$
\begin{aligned}
& \mathcal{N}_{11, k}^{(i)} \triangleq \mathbb{E}\left[\left(c \sum_{j=1}^{L} \bar{\zeta}_{k}^{(i j)} \Gamma \varepsilon_{k}^{(j)}\right)\left(c \sum_{j=1}^{L} \bar{\zeta}_{k}^{(i j)} \Gamma \varepsilon_{k}^{(j)}\right)^{T}\right], \\
& \mathcal{N}_{12, k}^{(i)} \triangleq \mathbb{E}\left[A_{k}^{(i)} \varepsilon_{k}^{(i)}\left(c \sum_{j=1}^{L} \bar{\zeta}_{k}^{(i j)} \Gamma \varepsilon_{k}^{(j)}\right)^{T}\right], \\
& \mathcal{N}_{22, k}^{(i)} \triangleq \mathbb{E}\left[\left(c \sum_{j=1}^{L} \tilde{\zeta}_{k}^{(i j)} \Gamma x_{k}^{(j)}\right)\left(c \sum_{j=1}^{L} \tilde{\zeta}_{k}^{(i j)} \Gamma x_{k}^{(j)}\right)^{T}\right], \\
& \tilde{\zeta}_{k}^{(i j)} \triangleq \zeta_{k}^{(i j)}-\bar{\zeta}_{k}^{(i j)} .
\end{aligned}
$$

Proof: Based on (1) and (7), we have

$$
\begin{aligned}
\varepsilon_{k+1 \mid k}^{(i)}= & A_{k}^{(i)} \varepsilon_{k}^{(i)}+c \sum_{j=1}^{L} \bar{\zeta}_{k}^{(i j)} \Gamma \varepsilon_{k}^{(j)} \\
& +c \sum_{j=1}^{L} \tilde{\zeta}_{k}^{(i j)} \Gamma x_{k}^{(j)}+B_{k}^{(i)} w_{k}^{(i)}
\end{aligned}
$$

The definition of $\Xi_{k+1 \mid k}^{(i)}$ tells

$$
\begin{aligned}
\Xi_{k+1 \mid k}^{(i)}= & A_{k}^{(i)} \Xi_{k}^{(i)}\left(A_{k}^{(i)}\right)^{T}+\mathcal{N}_{11, k}^{(i)}+\mathcal{N}_{22, k}^{(i)} \\
& +\mathcal{N}_{12, k}^{(i)}+\left(\mathcal{N}_{12, k}^{(i)}\right)^{T}+B_{k}^{(i)} Q_{k}^{(i)}\left(B_{k}^{(i)}\right)^{T} .
\end{aligned}
$$

In addition, we obtain

$$
\begin{aligned}
\mathcal{N}_{22, k}^{(i)} & =c^{2} \mathbb{E}\left[\left(\sum_{j=1}^{L} \tilde{\zeta}_{k}^{(i j)} \Gamma x_{k}^{(j)}\right)\left(\sum_{j=1}^{L} \tilde{\zeta}_{k}^{(i j)} \Gamma x_{k}^{(j)}\right)^{T}\right] \\
& =c^{2} \sum_{j=1}^{L} S_{k}^{(i j)} \Gamma O_{k}^{(j)} \Gamma^{T}
\end{aligned}
$$

where the fact that $\mathbb{E}\left[\tilde{\zeta}_{k}^{(i j)} \tilde{\zeta}_{k}^{(i m)}\right]=0(j \neq m)$ has been utilized. Substituting (18) into (17) yields (15).

Lemma 3: The recursion of $\Xi_{k+1}^{(i)}$ is

$$
\begin{aligned}
\Xi_{k+1}^{(i)}= & \Omega_{k+1}^{(i)} \Xi_{k+1 \mid k}^{(i)}\left(\Omega_{k+1}^{(i)}\right)^{T} \\
& +\beta_{k+1}^{(i)} G_{s, k+1}^{(i)} C_{k+1}^{(i)} \Xi_{k+1 \mid k}^{(i)}\left(C_{k+1}^{(i)}\right)^{T}\left(G_{s, k+1}^{(i)}\right)^{T} \\
& +\mathbb{E}\left[\tilde{G}_{k+1}^{(i)} \mu_{k+1}^{(i)}\left(\mu_{k+1}^{(i)}\right)^{T}\left(\tilde{G}_{k+1}^{(i)}\right)^{T}\right] \\
& +\mathbb{E}\left[\tilde{G}_{k+1}^{(i)} \varphi\left(C_{k+1}^{(i)} x_{k+1}^{(i)}\right) \varphi^{T}\left(C_{k+1}^{(i)} x_{k+1}^{(i)}\right)\left(\tilde{G}_{k+1}^{(i)}\right)^{T}\right] \\
& +G_{k+1}^{(i)}\left[R_{k+1}^{(i)}+C_{k+1}^{(i)} O_{k+1}^{(i)}\left(C_{k+1}^{(i)}\right)^{T}\right]\left(G_{k+1}^{(i)}\right)^{T} \\
& +\beta_{k+1}^{(i)} G_{s, k+1}^{(i)}\left[R_{k+1}^{(i)}+C_{k+1}^{(i)} O_{k+1}^{(i)}\left(C_{k+1}^{(i)}\right)^{T}\right]\left(G_{s, k+1}^{(i)}\right)^{T} \\
& +\operatorname{sym}\left\{\mathcal{H}_{1, k+1}^{(i)}+\mathcal{H}_{2, k+1}^{(i)}+\mathcal{H}_{3, k+1}^{(i)}+\mathcal{H}_{4, k+1}^{(i)}\right. \\
& \left.+\mathcal{H}_{5, k+1}^{(i)}+\mathcal{H}_{6, k+1}^{(i)}+\mathcal{H}_{7, k+1}^{(i)}\right\}
\end{aligned}
$$

where

$$
\begin{aligned}
\Omega_{k+1}^{(i)} & \triangleq I-G_{k+1}^{(i)} C_{k+1}^{(i)}, \tilde{\Omega}_{k+1}^{(i)} \triangleq I-\tilde{G}_{k+1}^{(i)} C_{k+1}^{(i)}, \\
\mathcal{H}_{1, k+1}^{(i)} & \triangleq \mathbb{E}\left[\tilde{\Omega}_{k+1}^{(i)} \varepsilon_{k+1 \mid k}^{(i)}\left(\mu_{k+1}^{(i)}\right)^{T}\left(\tilde{G}_{k+1}^{(i)}\right)^{T}\right], \\
\mathcal{H}_{2, k+1}^{(i)} & \triangleq \mathbb{E}\left[-\tilde{\Omega}_{k+1}^{(i)} \varepsilon_{k+1 \mid k}^{(i)} \varphi^{T}\left(C_{k+1}^{(i)} x_{k+1}^{(i)}\right)\left(\tilde{G}_{k+1}^{(i)}\right)^{T}\right], \\
\mathcal{H}_{3, k+1}^{(i)} & \triangleq \mathbb{E}\left[\tilde{\Omega}_{k+1}^{(i)} \varepsilon_{k+1 \mid k}^{(i)}\left(x_{k+1}^{(i)}\right)^{T}\left(C_{k+1}^{(i)}\right)^{T}\left(\tilde{G}_{k+1}^{(i)}\right)^{T}\right], \\
\mathcal{H}_{4, k+1}^{(i)} & \triangleq \mathbb{E}\left[-\tilde{G}_{k+1}^{(i)} \mu_{k+1}^{(i)} \varphi^{T}\left(C_{k+1}^{(i)} x_{k+1}^{(i)}\right)\left(\tilde{G}_{k+1}^{(i)}\right)^{T}\right], \\
\mathcal{H}_{5, k+1}^{(i)} & \triangleq \mathbb{E}\left[-\tilde{G}_{k+1}^{(i)} \mu_{k+1}^{(i)}\left(v_{k+1}^{(i)}\right)^{T}\left(\tilde{G}_{k+1}^{(i)}\right)^{T}\right], \\
\mathcal{H}_{6, k+1}^{(i)} & \triangleq \mathbb{E}\left[\tilde{G}_{k+1}^{(i)} \mu_{k+1}^{(i)}\left(x_{k+1}^{(i)}\right)^{T}\left(C_{k+1}^{(i)}\right)^{T}\left(\tilde{G}_{k+1}^{(i)}\right)^{T}\right], \\
\mathcal{H}_{7, k+1}^{(i)} & \triangleq \mathbb{E}\left[-\tilde{G}_{k+1}^{(i)} \varphi\left(C_{k+1}^{(i)} x_{k+1}^{(i)}\right)\left(C_{k+1}^{(i)} x_{k+1}^{(i)}\right)^{T}\left(\tilde{G}_{k+1}^{(i)}\right)^{T}\right] .
\end{aligned}
$$

Proof: Based on the definition of $\varepsilon_{k+1}^{(i)}$, we have

$$
\varepsilon_{k+1}^{(i)}=\varepsilon_{k+1 \mid k}^{(i)}-\tilde{G}_{k+1}^{(i)}\left(\tilde{z}_{k+1}^{(i)}-C_{k+1}^{(i)} \hat{x}_{k+1 \mid k}^{(i)}\right) .
$$

Adding two zero terms $z_{k+1}^{(i)}-z_{k+1}^{(i)}$ and $C_{k+1}^{(i)} x_{k+1}^{(i)}-$ $C_{k+1}^{(i)} x_{k+1}^{(i)}$ to the right-hand side of (20) yields

$$
\begin{aligned}
\varepsilon_{k+1}^{(i)}= & \tilde{\Omega}_{k+1}^{(i)} \varepsilon_{k+1 \mid k}^{(i)}+\tilde{G}_{k+1}^{(i)} \mu_{k+1}^{(i)} \\
& -\tilde{G}_{k+1}^{(i)} \varphi\left(C_{k+1}^{(i)} x_{k+1}^{(i)}\right)-\tilde{G}_{k+1}^{(i)} v_{k+1}^{(i)} \\
& +\tilde{G}_{k+1}^{(i)} C_{k+1}^{(i)} x_{k+1}^{(i)} .
\end{aligned}
$$

We know

$$
\mathbb{E}\left[\tilde{\Omega}_{k+1}^{(i)} \varepsilon_{k+1 \mid k}^{(i)}\left(\varepsilon_{k+1 \mid k}^{(i)}\right)^{T}\left(\tilde{\Omega}_{k+1}^{(i)}\right)^{T}\right]
$$




$$
\begin{aligned}
= & \Omega_{k+1}^{(i)} \Xi_{k+1 \mid k}^{(i)}\left(\Omega_{k+1}^{(i)}\right)^{T} \\
& +\beta_{k+1}^{(i)} G_{s, k+1}^{(i)} C_{k+1}^{(i)} \Xi_{k+1 \mid k}^{(i)}\left(C_{k+1}^{(i)}\right)^{T}\left(G_{s, k+1}^{(i)}\right)^{T}, \\
& \mathbb{E}\left[\tilde{G}_{k+1}^{(i)} v_{k+1}^{(i)}\left(v_{k+1}^{(i)}\right)^{T}\left(\tilde{G}_{k+1}^{(i)}\right)^{T}\right] \\
= & G_{k+1}^{(i)} R_{k+1}^{(i)}\left(G_{k+1}^{(i)}\right)^{T}+\beta_{k+1}^{(i)} G_{s, k+1}^{(i)} R_{k+1}^{(i)}\left(G_{s, k+1}^{(i)}\right)^{T}, \\
& \mathbb{E}\left[\tilde{G}_{k+1}^{(i)}\left(C_{k+1}^{(i)} x_{k+1}^{(i)}\right)\left(C_{k+1}^{(i)} x_{k+1}^{(i)}\right)^{T}\left(\tilde{G}_{k+1}^{(i)}\right)^{T}\right] \\
= & G_{k+1}^{(i)} C_{k+1}^{(i)} O_{k+1}^{(i)}\left(C_{k+1}^{(i)}\right)^{T}\left(G_{k+1}^{(i)}\right)^{T} \\
& +\beta_{k+1}^{(i)} G_{s, k+1}^{(i)} C_{k+1}^{(i)} O_{k+1}^{(i)}\left(C_{k+1}^{(i)}\right)^{T}\left(G_{s, k+1}^{(i)}\right)^{T} .
\end{aligned}
$$

Noting that $\varepsilon_{k+1 \mid k}^{(i)}$ and $x_{k+1}^{(i)}$ are both uncorrelated with $v_{k+1}^{(i)}$, we have

$$
\begin{aligned}
& \mathbb{E}\left[-\tilde{\Omega}_{k+1}^{(i)} \varepsilon_{k+1 \mid k}^{(i)}\left(v_{k+1}^{(i)}\right)^{T}\left(\tilde{G}_{k+1}^{(i)}\right)^{T}\right]=0, \\
& \mathbb{E}\left[\tilde{G}_{k+1}^{(i)} \varphi^{T}\left(C_{k+1}^{(i)} x_{k+1}^{(i)}\right)\left(v_{k+1}^{(i)}\right)^{T}\left(\tilde{G}_{k+1}^{(i)}\right)^{T}\right]=0, \\
& \mathbb{E}\left[-\tilde{G}_{k+1}^{(i)} v_{k+1}^{(i)}\left(C_{k+1}^{(i)} x_{k+1}^{(i)}\right)^{T}\left(\tilde{G}_{k+1}^{(i)}\right)^{T}\right]=0 .
\end{aligned}
$$

Based on the definition of $\Xi_{k+1}^{(i)}$ and (21)-(25), $\Xi_{k+1}^{(i)}$ can be calculated via (19).

So far, we have made much effort to obtain the expressions of error covariance (as shown in Lemmas 2-3) with sensor saturations, switching topologies, DECP and SGVS. Unfortunately, it is hard to compute their accurate values due to the existence of the unknown terms $\mathcal{N}_{12, k}^{(i)}$ and $\mathcal{H}_{j, k+1}^{(i)},(j=$ $1,2, \cdots, 7)$. In search of an alternative scheme, the upperbounding technique seems a feasible solution, that is, we like to find an upper bound on the error covariance by virtue of the recursive difference equation approach.

Theorem 1: Let $\pi_{i}, \alpha_{i}$ and $\beta_{i}(i=2, \cdots, 5)$ be positive scalars. Under the initial condition $\bar{\Xi}_{0}^{(i)}=\Xi_{0}^{(i)}>0$, assume that there exist solutions $\bar{\Xi}_{k+1 \mid k}^{(i)}$ and $\Xi_{k+1}^{(i)}$ to

$$
\begin{aligned}
\bar{\Xi}_{k+1 \mid k}^{(i)}= & \left(1+\pi_{2}\right) A_{k}^{(i)} \bar{\Xi}_{k}^{(i)}\left(A_{k}^{(i)}\right)^{T} \\
& +\left(1+\pi_{2}^{-1}\right) c^{2} M_{i} \sum_{j=1}^{L} \bar{\zeta}_{k}^{(i j)} \Gamma \bar{\Xi}_{k}^{(j)} \Gamma^{T} \\
& +c^{2} \sum_{j=1}^{L} S_{k}^{(i j)} \Gamma \bar{O}_{k}^{(j)} \Gamma^{T}+B_{k}^{(i)} Q_{k}^{(i)}\left(B_{k}^{(i)}\right)^{T}
\end{aligned}
$$

and

$$
\begin{aligned}
\bar{\Xi}_{k+1}^{(i)}= & \left(1+\pi_{3}+\pi_{4}+\pi_{5}\right)\left[\Omega_{k+1}^{(i)} \bar{\Xi}_{k+1 \mid k}^{(i)}\left(\Omega_{k+1}^{(i)}\right)^{T}\right. \\
& \left.+\beta_{k+1}^{(i)} G_{s, k+1}^{(i)} C_{k+1}^{(i)} \bar{\Xi}_{k+1 \mid k}^{(i)}\left(C_{k+1}^{(i)}\right)^{T}\left(G_{s, k+1}^{(i)}\right)^{T}\right] \\
& +G_{k+1}^{(i)} \bar{U}_{k+1}^{(i)}\left(G_{k+1}^{(i)}\right)^{T}+\beta_{k+1}^{(i)} G_{s, k+1}^{(i)} \bar{U}_{k+1}^{(i)}\left(G_{s, k+1}^{(i)}\right)^{T}
\end{aligned}
$$

where

$$
\begin{aligned}
\bar{X}_{0}^{(i)}= & \left(\chi_{0}^{(i)}\right)^{2}, \bar{\rho} \triangleq \sum_{j=1}^{m} \rho_{j}^{2}, \\
\bar{X}_{k+1}^{(i)}= & {\left[\left(1+\alpha_{i}\right)\left(1+\iota_{i}\right) h_{i}^{2}+\frac{\left(1+\theta_{i}\right)}{\theta_{i}^{2}}\right.} \\
& \left.\times\left(1+\alpha_{i}^{-1}\right)\right] \bar{X}_{k}^{(i)}+\left[\left(1+\alpha_{i}\right)\left(1+\iota_{i}^{-1}\right)\right. \\
& \left.+\left(1+\alpha_{i}^{-1}\right)\left(1+\theta_{i}^{-1}\right)\right] \sigma_{i}^{2},
\end{aligned}
$$

$$
\begin{aligned}
\phi_{k+1}^{(i)} \triangleq & \left(1+\theta_{i}\right) \frac{\bar{X}_{k+1}^{(i)}}{\theta_{i}^{2}}+\left(1+\theta_{i}^{-1}\right) \sigma_{i}^{2}, \\
U_{k+1}^{(i)} \triangleq & \left(1+\pi_{3}^{-1}+\pi_{6}+\pi_{7}+\pi_{8}\right) \phi_{k+1}^{(i)} I \\
& +\left(1+\pi_{4}^{-1}+\pi_{6}^{-1}+\pi_{9}\right) \bar{\rho} I+\left(1+\pi_{7}^{-1}\right) R_{k+1}^{(i)} \\
& +\left(1+\pi_{5}^{-1}+\pi_{8}^{-1}+\pi_{9}^{-1}\right) C_{k+1}^{(i)} O_{k+1}^{(i)}\left(C_{k+1}^{(i)}\right)^{T},
\end{aligned}
$$

and $\bar{U}_{k+1}^{(i)}$ is the matrix obtained by replacing $O_{k+1}^{(i)}$ by $\bar{O}_{k+1}^{(i)}$ in $U_{k+1}^{(i)}$. Then, the inequalities $\Xi_{k+1 \mid k}^{(i)} \leq \Xi_{k+1 \mid k}^{(i)}$ and $\Xi_{k+1}^{(i)} \leq$ $\bar{\Xi}_{k+1}^{(i)}$ always hold.

Proof: With initial conditions, we have $\Xi_{0}^{(i)} \leq \bar{\Xi}_{0}^{(i)}$. Assuming that $\Xi_{k}^{(i)} \leq \bar{\Xi}_{k}^{(i)}$, we need to prove that $\Xi_{k+1}^{(i)} \leq \bar{\Xi}_{k+1}^{(i)}$.

Similar to (12), we have

$$
\begin{aligned}
\mathcal{N}_{11, k}^{(i)} & =c^{2} \sum_{j=1}^{L} \sum_{m=1}^{L} \bar{\zeta}_{k}^{(i j)} \bar{\zeta}_{k}^{(i m)} \Gamma \mathbb{E}\left[\varepsilon_{k}^{(j)}\left(\varepsilon_{k}^{(m)}\right)^{T}\right] \Gamma^{T} \\
& \leq c^{2} M_{i} \sum_{j=1}^{L} \bar{\zeta}_{k}^{(i j)} \Gamma \Xi_{k}^{(j)} \Gamma^{T} .
\end{aligned}
$$

Next, let us handle the term $\mathcal{N}_{12, k}^{(i)}+\left(\mathcal{N}_{12, k}^{(i)}\right)^{T}$ in (15). By utilizing (11), one has

$$
\mathcal{N}_{12, k}^{(i)}+\left(\mathcal{N}_{12, k}^{(i)}\right)^{T} \leq \pi_{2} A_{k}^{(i)} \Xi_{k}^{(i)}\left(A_{k}^{(i)}\right)^{T}+\pi_{2}^{-1} \mathcal{N}_{11, k}^{(i)}
$$

Substituting (29) into (15) leads to

$$
\begin{aligned}
\Xi_{k+1 \mid k}^{(i)} \leq & \left(1+\pi_{2}\right) A_{k}^{(i)} \Xi_{k}^{(i)}\left(A_{k}^{(i)}\right)^{T} \\
& +\left(1+\pi_{2}^{-1}\right) c^{2} M_{i} \sum_{j=1}^{L} \bar{\zeta}_{k}^{(i j)} \Gamma \Xi_{k}^{(j)} \Gamma^{T} \\
& +c^{2} \sum_{j=1}^{L} S_{k}^{(i j)} \Gamma O_{k}^{(j)} \Gamma^{T}+B_{k}^{(i)} Q_{k}^{(i)}\left(B_{k}^{(i)}\right)^{T} .
\end{aligned}
$$

Then, from Lemma 1 and the assumption that $\Xi_{k}^{(i)} \leq \bar{\Xi}_{k}^{(i)}$, it is easy to show that $\Xi_{k+1 \mid k}^{(i)} \leq \bar{\Xi}_{k+1 \mid k}^{(i)}$.

In view of (3)-(4) and (11), we obtain

$$
\left(\mu_{k+1}^{(i)}\right)^{T} \mu_{k+1}^{(i)} \leq\left(1+\theta_{i}\right) \frac{\left(\chi_{k+1}^{(i)}\right)^{2}}{\theta_{i}^{2}}+\left(1+\theta_{i}^{-1}\right) \sigma_{i}^{2}
$$

and

$$
\begin{aligned}
& \mathbb{E}\left[\left(\chi_{k+1}^{(i)}\right)^{2}\right] \\
= & \mathbb{E}\left[\left(h_{i} \chi_{k}^{(i)}+\sigma_{i}-\left\|\mu_{k}^{(i)}\right\|\right)^{2}\right] \\
\leq & \mathbb{E}\left[\left(1+\alpha_{i}\right)\left(h_{i} \chi_{k}^{(i)}+\sigma_{i}\right)^{2}+\left(1+\alpha_{i}^{-1}\right)\left\|\mu_{k}^{(i)}\right\|^{2}\right] \\
\leq & \left(1+\alpha_{i}\right)\left[\left(1+\iota_{i}\right) h_{i}^{2} \mathbb{E}\left(\chi_{k}^{(i)}\right)^{2}+\left(1+\iota_{i}^{-1}\right) \sigma_{i}^{2}\right] \\
& \left.+\left(1+\alpha_{i}^{-1}\right) \mathbb{E}\left\|\mu_{k}^{(i)}\right\|^{2}\right] .
\end{aligned}
$$

Based on Lemma 4 in [19], we observe that $\mathbb{E}\left[\left(\chi_{k+1}^{(i)}\right)^{2}\right] \leq \bar{X}_{k+1}^{(i)}$. Hence, it is not difficult to verify that $\mathbb{E}\left[\mu_{k+1}^{(i)}\left(\mu_{k+1}^{(i)}\right)^{T}\right] \leq \phi_{k+1}^{(i)} I$. Furthermore, we have

$$
\begin{aligned}
& \mathbb{E}\left[\tilde{G}_{k+1}^{(i)} \mu_{k+1}^{(i)}\left(\mu_{k+1}^{(i)}\right)^{T}\left(\tilde{G}_{k+1}^{(i)}\right)^{T}\right] \\
= & G_{k+1}^{(i)} \mathbb{E}\left[\mu_{k+1}^{(i)}\left(\mu_{k+1}^{(i)}\right)^{T}\right]\left(G_{k+1}^{(i)}\right)^{T} \\
& +\beta_{k+1}^{(i)} G_{s, k+1}^{(i)} \mathbb{E}\left[\mu_{k+1}^{(i)}\left(\mu_{k+1}^{(i)}\right)^{T}\right]\left(G_{s, k+1}^{(i)}\right)^{T}
\end{aligned}
$$




$$
\leq G_{k+1}^{(i)} \phi_{k+1}^{(i)}\left(G_{k+1}^{(i)}\right)^{T}+\beta_{k+1}^{(i)} G_{s, k+1}^{(i)} \phi_{k+1}^{(i)}\left(G_{s, k+1}^{(i)}\right)^{T},
$$

and

$$
\begin{aligned}
& \mathbb{E}\left[\tilde{G}_{k+1}^{(i)} \varphi\left(C_{k+1}^{(i)} x_{k+1}^{(i)}\right) \varphi^{T}\left(C_{k+1}^{(i)} x_{k+1}^{(i)}\right)\left(\tilde{G}_{k+1}^{(i)}\right)^{T}\right] \\
\leq & \bar{\rho} G_{k+1}^{(i)}\left(G_{k+1}^{(i)}\right)^{T}+\beta_{k+1}^{(i)} \bar{\rho} G_{s, k+1}^{(i)}\left(G_{s, k+1}^{(i)}\right)^{T} .
\end{aligned}
$$

Similar to (29), the last term of the right-hand side of (19) is computed as

$$
\begin{aligned}
& \mathcal{H}_{1, k+1}^{(i)}+\left(\mathcal{H}_{1, k+1}^{(i)}\right)^{T} \\
\leq & \pi_{3} \mathbb{E}\left[\tilde{\Omega}_{k+1}^{(i)} \varepsilon_{k+1 \mid k}^{(i)}\left(\varepsilon_{k+1 \mid k}^{(i)}\right)^{T}\left(\tilde{\Omega}_{k+1}^{(i)}\right)^{T}\right] \\
& +\pi_{3}^{-1} \mathbb{E}\left[\tilde{G}_{k+1}^{(i)} \mu_{k+1}^{(i)}\left(\mu_{k+1}^{(i)}\right)^{T}\left(\tilde{G}_{k+1}^{(i)}\right)^{T}\right] \\
& \mathcal{H}_{2, k+1}^{(i)}+\left(\mathcal{H}_{2, k+1}^{(i)}\right)^{T} \\
\leq & \pi_{4} \mathbb{E}\left[\tilde{\Omega}_{k+1}^{(i)} \varepsilon_{k+1 \mid k}^{(i)}\left(\varepsilon_{k+1 \mid k}^{(i)}\right)^{T}\left(\tilde{\Omega}_{k+1}^{(i)}\right)^{T}\right] \\
& +\pi_{4}^{-1} \mathbb{E}\left[\tilde{G}_{k+1}^{(i)} \varphi\left(C_{k+1}^{(i)} x_{k+1}^{(i)}\right) \varphi^{T}\left(C_{k+1}^{(i)} x_{k+1}^{(i)}\right)\left(\tilde{G}_{k+1}^{(i)}\right)^{T}\right] \\
& \mathcal{H}_{3, k+1}^{(i)}+\left(\mathcal{H}_{3, k+1}^{(i)}\right)^{T} \\
\leq & \pi_{5} \mathbb{E}\left[\tilde{\Omega}_{k+1}^{(i)} \varepsilon_{k+1 \mid k}^{(i)}\left(\varepsilon_{k+1 \mid k}^{(i)}\right)^{T}\left(\tilde{\Omega}_{k+1}^{(i)}\right)^{T}\right] \\
& +\pi_{5}^{-1} \mathbb{E}\left[\tilde{G}_{k+1}^{(i)} C_{k+1}^{(i)} x_{k+1}^{(i)}\left(C_{k+1}^{(i)} x_{k+1}^{(i)}\right)^{T}\left(\tilde{G}_{k+1}^{(i)}\right)^{T}\right] \\
& \mathcal{H}_{4, k+1}^{(i)}+\left(\mathcal{H}_{4, k+1}^{(i)}\right)^{T} \\
\leq & \pi_{6} \mathbb{E}\left[\tilde{G}_{k+1}^{(i)} \mu_{k+1}^{(i)}\left(\mu_{k+1}^{(i)}\right)^{T}\left(\tilde{G}_{k+1}^{(i)}\right)^{T}\right] \\
& +\pi_{6}^{-1} \mathbb{E}\left[\tilde{G}_{k+1}^{(i)} \varphi\left(C_{k+1}^{(i)} x_{k+1}^{(i)}\right) \varphi^{T}\left(C_{k+1}^{(i)} x_{k+1}^{(i)}\right)\left(\tilde{G}_{k+1}^{(i)}\right)^{T}\right], \\
& \mathcal{H}_{5, k+1}^{(i)}+\left(\mathcal{H}_{5, k+1}^{(i)}\right)^{T} \\
\leq & \pi_{7} \mathbb{E}\left[\tilde{G}_{k+1}^{(i)} \mu_{k+1}^{(i)}\left(\mu_{k+1}^{(i)}\right)^{T}\left(\tilde{G}_{k+1}^{(i)}\right)^{T}\right] \\
& +\pi_{7}^{-1} \mathbb{E}\left[\tilde{G}_{k+1}^{(i)} v_{k+1}^{(i)}\left(v_{k+1}^{(i)}\right)^{T}\left(\tilde{G}_{k+1}^{(i)}\right)^{T}\right] \\
& \mathcal{H}_{6, k+1}^{(i)}+\left(\mathcal{H}_{6, k+1}^{(i)}\right)^{T} \\
\leq & \pi_{8} \mathbb{E}\left[\tilde{G}_{k+1}^{(i)} \mu_{k+1}^{(i)}\left(\mu_{k+1}^{(i)}\right)^{T}\left(\tilde{G}_{k+1}^{(i)}\right)^{T}\right] \\
& +\pi_{8}^{-1} \mathbb{E}\left[\tilde{G}_{k+1}^{(i)}\left(C_{k+1}^{(i)} x_{k+1}^{(i)}\right)\left(C_{k+1}^{(i)} x_{k+1}^{(i)}\right)^{T}\left(\tilde{G}_{k+1}^{(i)}\right)^{T}\right] \\
& \mathcal{H}_{7, k+1}^{(i)}+\left(\mathcal{H}_{7, k+1}^{(i)}\right)^{T} \\
\leq & \pi_{9} \mathbb{E}\left[\tilde{G}_{k+1}^{(i)} \varphi\left(C_{k+1}^{(i)} x_{k+1}^{(i)}\right) \varphi^{T}\left(C_{k+1}^{(i)} x_{k+1}^{(i)}\right)\left(\tilde{G}_{k+1}^{(i)}\right)^{T}\right] \\
& +\pi_{9}^{-1} \mathbb{E}\left[\tilde{G}_{k+1}^{(i)}\left(C_{k+1}^{(i)} x_{k+1}^{(i)}\right)\left(C_{k+1}^{(i)} x_{k+1}^{(i)}\right)^{T}\left(\tilde{G}_{k+1}^{(i)}\right)^{T}\right]
\end{aligned}
$$

Next, substituting (33)-(41) into (19) yields

$$
\begin{aligned}
\Xi_{k+1}^{(i)} \leq & \left(1+\pi_{3}+\pi_{4}+\pi_{5}\right)\left[\Omega_{k+1}^{(i)} \Xi_{k+1 \mid k}^{(i)}\left(\Omega_{k+1}^{(i)}\right)^{T}\right. \\
& \left.+\beta_{k+1}^{(i)} G_{s, k+1}^{(i)} C_{k+1}^{(i)} \Xi_{k+1 \mid k}^{(i)}\left(C_{k+1}^{(i)}\right)^{T}\left(G_{s, k+1}^{(i)}\right)^{T}\right] \\
& +G_{k+1}^{(i)} U_{k+1}^{(i)}\left(G_{k+1}^{(i)}\right)^{T}+\beta_{k+1}^{(i)} G_{s, k+1}^{(i)} U_{k+1}^{(i)}\left(G_{s, k+1}^{(i)}\right)^{T} .
\end{aligned}
$$

Using mathematical induction, we have $\Xi_{k+1}^{(i)} \leq \bar{\Xi}_{k+1}^{(i)}$.

Theorem 2: Consider the discrete CNs with sensor saturations described by (1) and the proposed non-fragile filter given by (7). The upper bound on $\bar{\Xi}_{k+1}^{(i)}$ is minimized with parameter

$$
\begin{aligned}
G_{k+1}^{(i)}= & \left(1+\pi_{3}+\pi_{4}+\pi_{5}\right) \bar{\Xi}_{k+1 \mid k}^{(i)}\left(C_{k+1}^{(i)}\right)^{T}\left[\left(1+\pi_{3}\right.\right. \\
& \left.\left.+\pi_{4}+\pi_{5}\right) C_{k+1}^{(i)} \bar{\Xi}_{k+1 \mid k}^{(i)}\left(C_{k+1}^{(i)}\right)^{T}+\bar{U}_{k+1}^{(i)}\right]^{-1} .
\end{aligned}
$$

Proof: Taking partial derivative of $\operatorname{tr}\left(\bar{\Xi}_{k+1}^{(i)}\right)$ with respect to the parameters $G_{k+1}^{(i)}$ and letting the partial derivative be zero, we obtain

$$
\begin{aligned}
& \frac{\partial \operatorname{tr}\left(\bar{\Xi}_{k+1}^{(i)}\right)}{\partial G_{k+1}^{(i)}} \\
= & -2\left(1+\pi_{3}+\pi_{4}+\pi_{5}\right) \bar{\Xi}_{k+1 \mid k}^{(i)}\left(C_{k+1}^{(i)}\right)^{T} \\
& +2\left(1+\pi_{3}+\pi_{4}+\pi_{5}\right) G_{k+1}^{(i)} C_{k+1}^{(i)} \bar{\Xi}_{k+1 \mid k}^{(i)}\left(C_{k+1}^{(i)}\right)^{T} \\
& +2 G_{k+1}^{(i)} \bar{U}_{k+1}^{(i)} \\
= & 0 .
\end{aligned}
$$

After some algebraic manipulations, we have

$$
\begin{aligned}
& G_{k+1}^{(i)}\left[\left(1+\pi_{3}+\pi_{4}+\pi_{5}\right) C_{k+1}^{(i)} \bar{\Xi}_{k+1 \mid k}^{(i)}\left(C_{k+1}^{(i)}\right)^{T}+\bar{U}_{k+1}^{(i)}\right] \\
= & \left(1+\pi_{3}+\pi_{4}+\pi_{5}\right) \bar{\Xi}_{k+1 \mid k}^{(i)}\left(C_{k+1}^{(i)}\right)^{T},
\end{aligned}
$$

which yields (43).

Remark 4: So far, we have handled the dynamic eventtriggered non-fragile filtering issue for $\mathrm{CNs}$ with sensor saturations and switching topologies. It should be pointed out that the factors of sensor saturations, switching topologies, DECP and SGVs have brought some essential difficulties in the design of the filtering scheme. Sufficient conditions that guarantee the existence of the filter have been established via Theorems 1-2, where the effects from the above-mentioned factors on filter performance have been considered. More specifically, $\bar{\rho}$ is related to the saturation levels, $M_{i}$ accounts for the switching topologies, $\beta_{k+1}^{(i)}$ stands for the covariance of the multiplicative noise and $\phi_{k+1}^{(i)}$ is there for the DECP. It is also worthwhile to notice that the proposed dynamic event-triggered non-fragile filtering algorithm is of a recursive characteristic facilitating online implementations.

\section{B. Analysis of Boundedness of Filtering Errors}

To facilitate further developments, the following definition and assumption are first introduced.

Definition 1: [17] $\varsigma_{k}$ is exponentially mean-square bounded if there exist positive numbers $\tau, u$ and $0<\alpha<1$ such that

$$
\mathbb{E}\left\{\left\|\varsigma_{k}\right\|^{2}\right\} \leq \tau \mathbb{E}\left\{\left\|\varsigma_{0}\right\|^{2}\right\} \alpha^{k}+u .
$$

Assumption 1: There exist positive real scalars $\bar{a}, \bar{b}, \underline{b}, \underline{c}, \bar{c}$, $\bar{k}_{s}, \bar{t}, \bar{r}, \bar{v}_{x}, \bar{q}, \underline{q}, \bar{d}, \bar{\zeta}, \bar{s}, \bar{\beta}$ and $\bar{\phi}$, such that the following conditions hold

$$
\begin{aligned}
& \left\|A_{k}^{(i)}\right\| \leq \bar{a}, \underline{b} I \leq B_{k}^{(i)}\left(B_{k}^{(i)}\right)^{T} \leq \bar{b} I, \\
& \underline{c} \leq\left\|C_{k+1}^{(i)}\right\| \leq \bar{c},\left\|G_{s, k+1}^{(i)}\right\| \leq \bar{k}_{s}, \\
& \left\|M_{i}\right\| \leq \bar{t},\|\Gamma\| \leq \bar{r},\left\|\operatorname{tr}\left(\bar{O}_{k}^{(i)}\right)\right\| \leq \bar{v}_{x}, \\
& \underline{q} I \leq Q_{k}^{(i)} \leq \bar{q} I,\left\|R_{k}^{(i)}\right\| \leq \bar{d},\left\|\bar{\zeta}_{k}^{(i j)}\right\| \leq \bar{\zeta}, \\
& \left\|S_{k}^{(i j)}\right\| \leq \bar{s},\left\|\beta_{k+1}^{(i)}\right\| \leq \bar{\beta},\left\|\phi_{k+1}^{(i)}\right\| \leq \bar{\phi} .
\end{aligned}
$$

For convenience of later analysis, we denote

$$
\begin{aligned}
\Pi_{k}^{(i)} & \triangleq \operatorname{diag}_{L}\left\{\Omega_{k}^{(i)}\right\}, \\
\Pi_{k} & \triangleq \operatorname{diag}\left\{\Omega_{k}^{(1)}, \Omega_{k}^{(2)}, \cdots, \Omega_{k}^{(L)}\right\},
\end{aligned}
$$




$$
\begin{aligned}
& \tilde{A}_{k} \triangleq \operatorname{diag}\left\{A_{k}^{(1)}, A_{k}^{(2)}, \cdots, A_{k}^{(L)}\right\}, \\
& \tilde{B}_{k} \triangleq \operatorname{diag}\left\{B_{k}^{(1)}, B_{k}^{(2)}, \cdots, B_{k}^{(L)}\right\}, \\
& \tilde{C}_{k} \triangleq \operatorname{diag}\left\{C_{k}^{(1)}, C_{k}^{(2)}, \cdots, C_{k}^{(L)}\right\}, \\
& \tilde{Q}_{k} \triangleq \operatorname{diag}\left\{Q_{k}^{(1)}, Q_{k}^{(2)}, \cdots, Q_{k}^{(L)}\right\}, \\
& \tilde{G}_{s, k} \triangleq \operatorname{diag}\left\{G_{s, k}^{(1)}, G_{s, k}^{(2)}, \cdots, G_{s, k}^{(L)}\right\}, \\
& \tilde{M}_{i} \triangleq \operatorname{diag}_{L}\left\{M_{i}\right\}, \tilde{\Gamma}^{(L)} \operatorname{diag}_{L}\{\Gamma\}, \\
& \tilde{G}_{s, k}^{(i)} \triangleq \operatorname{diag}_{L}\left\{G_{s, k}^{(i)}\right\}, \tilde{C}_{k}^{(i)} \triangleq \operatorname{diag}_{L}\left\{C_{k}^{(i)}\right\} \\
& \mathcal{I} \triangleq[I, I, \cdots, I], \mathcal{O} \triangleq[0,0, \cdots, 0], \\
& E_{i} \triangleq \operatorname{col}_{L}\{\mathcal{O}, \cdots, \mathcal{O}, \mathcal{I}, \mathcal{O}, \cdots, \mathcal{O}\}, \\
& \Delta_{k}^{(i)} \triangleq \operatorname{diag}_{k}\left\{\bar{\zeta}_{k}^{(i 1)}, \bar{\zeta}_{k}^{(i 2)}, \cdots, \bar{\zeta}_{k}^{(i L)}\right\}, \\
& \bar{e}_{i} \triangleq[\underbrace{0,0, \cdots, 0, I, \underbrace{0, \cdots, 0}_{L-i}}_{i-1}], \mathscr{E} \triangleq \operatorname{diag}_{L}\{b I\} .
\end{aligned}
$$

Theorem 3: Consider the discrete CNs with sensor saturations described by (1) and the proposed non-fragile filter given by (7). Under Assumption 1, if the following two inequalities

$$
\delta_{1} \triangleq\left(\bar{m}^{2}+\bar{k}_{s}^{2} \bar{c}^{2}\right)\left(\bar{a}^{2}+L^{2} \bar{t} \bar{\zeta} \bar{r}^{2}\right)<1
$$

and

$$
\begin{aligned}
\nu \triangleq & \left(1+\eta_{4}+\eta_{5}\right)(1+\bar{f}+\bar{\beta}) \\
& +\left(1+\eta_{4}^{-1}+\eta_{6}\right) c^{2} L(1+\bar{\beta})<1
\end{aligned}
$$

hold, then $\varepsilon_{k}^{(i)}$ is exponentially mean-square bounded.

Proof: From (16) and (21), we have

$$
\begin{aligned}
\varepsilon_{k+1}^{(i)}= & \tilde{\Omega}_{k+1}^{(i)} A_{k}^{(i)} \varepsilon_{k}^{(i)}+\tilde{\Omega}_{k+1}^{(i)} c \sum_{j=1}^{L} \bar{\zeta}_{k}^{(i j)} \Gamma \varepsilon_{k}^{(j)} \\
& +\gamma_{k+1}^{(i)}+g_{k+1}^{(i)}
\end{aligned}
$$

where

$$
\begin{aligned}
\gamma_{k+1}^{(i)} \triangleq & \tilde{\Omega}_{k+1}^{(i)} c \sum_{j=1}^{L} \tilde{\zeta}_{k}^{(i j)} \Gamma x_{k}^{(j)}+\tilde{\Omega}_{k+1}^{(i)} B_{k}^{(i)} w_{k}^{(i)}-\tilde{G}_{k+1}^{(i)} v_{k+1}^{(i)} \\
g_{k+1}^{(i)} \triangleq & \tilde{G}_{k+1}^{(i)} \mu_{k+1}^{(i)}-\tilde{G}_{k+1}^{(i)} \varphi\left(C_{k+1}^{(i)} x_{k+1}^{(i)}\right) \\
& +\tilde{G}_{k+1}^{(i)} C_{k+1}^{(i)} x_{k+1}^{(i)} .
\end{aligned}
$$
that

Recalling the definition of $\Omega_{k+1}^{(i)}$ and (43), it can be deduced

$$
\begin{aligned}
& \left\|G_{k+1}^{(i)}\right\| \leq \frac{\bar{c}}{\underline{c}^{2}} \triangleq \bar{k} \\
& \left\|\Omega_{k+1}^{(i)}\right\| \leq 1+\frac{\bar{c}^{2}}{\underline{c}^{2}} \triangleq \bar{m} .
\end{aligned}
$$

Next, it follows from the definitions of $\gamma_{k+1}^{(i)}$ and $g_{k+1}^{(i)}$ in (50) that

$$
\begin{aligned}
& \mathbb{E}\left[\left(\gamma_{k+1}^{(i)}\right)^{T} \gamma_{k+1}^{(i)}\right] \\
= & c^{2} \sum_{j=1}^{L} S_{k}^{(i j)} \mathbb{E}\left[\left(x_{k}^{(j)}\right)^{T} \Gamma^{T} \Lambda_{k+1}^{(i)} \Gamma x_{k}^{(j)}\right] \\
& +\mathbb{E}\left[\left(w_{k}^{(i)}\right)^{T}\left(B_{k}^{(i)}\right)^{T} \Lambda_{k+1}^{(i)} B_{k}^{(i)} w_{k}^{(i)}\right] \\
& +\mathbb{E}\left[\left(v_{k+1}^{(i)}\right)^{T}\left(\tilde{G}_{k+1}^{(i)}\right)^{T} \tilde{G}_{k+1}^{(i)} v_{k+1}^{(i)}\right]
\end{aligned}
$$

$$
\begin{aligned}
& \leq c^{2} \sum_{j=1}^{L} S_{k}^{(i j)} \operatorname{tr} \mathbb{E}\left\{\left[x_{k}^{(j)}\left(x_{k}^{(j)}\right)^{T}\right] \Gamma^{T} \Lambda_{k+1}^{(i)} \Gamma\right\} \\
& +\operatorname{tr} \mathbb{E}\left\{w_{k}^{(i)}\left(w_{k}^{(i)}\right)^{T}\left(B_{k}^{(i)}\right)^{T} \Lambda_{k+1}^{(i)} B_{k}^{(i)}\right\} \\
& \quad+\operatorname{tr} \mathbb{E}\left\{v_{k+1}^{(i)}\left(v_{k+1}^{(i)}\right)^{T}\left(\tilde{G}_{k+1}^{(i)}\right)^{T} \tilde{G}_{k+1}^{(i)}\right\} \\
& \leq \\
& \left(\bar{m}^{2}+\bar{\beta} \bar{c}^{2} \bar{k}_{s}^{2}\right)\left(c^{2} L \bar{s} \bar{v}_{x} \bar{r}^{2}+n \bar{q} \bar{b}^{2}\right) \\
& \quad+m \bar{d}\left(\bar{k}^{2}+\bar{\beta} \bar{k}_{s}^{2}\right) \triangleq \bar{\gamma}
\end{aligned}
$$

and

$$
\begin{aligned}
\mathbb{E} & {\left[\left(g_{k+1}^{(i)}\right)^{T} g_{k+1}^{(i)}\right] } \\
= & \mathbb{E}\left[\left(\mu_{k+1}^{(i)}\right)^{T}\left(\tilde{G}_{k+1}^{(i)}\right)^{T} \tilde{G}_{k+1}^{(i)} \mu_{k+1}^{(i)}\right] \\
& +\mathbb{E}\left[\varphi^{T}\left(C_{k+1}^{(i)} x_{k+1}^{(i)}\right)\left(\tilde{G}_{k+1}^{(i)}\right)^{T} \tilde{G}_{k+1}^{(i)} \varphi\left(C_{k+1}^{(i)} x_{k+1}^{(i)}\right)\right] \\
& +\mathbb{E}\left[\left(x_{k+1}^{(i)}\right)^{T}\left(C_{k+1}^{(i)}\right)^{T}\left(\tilde{G}_{k+1}^{(i)}\right)^{T} \tilde{G}_{k+1}^{(i)} C_{k+1}^{(i)} x_{k+1}^{(i)}\right] \\
& +\operatorname{sym}\left\{\mathbb{E}\left[-\left(\mu_{k+1}^{(i)}\right)^{T}\left(\tilde{G}_{k+1}^{(i)}\right)^{T} \tilde{G}_{k+1}^{(i)} \varphi\left(C_{k+1}^{(i)} x_{k+1}^{(i)}\right)\right]\right. \\
& +\mathbb{E}\left[\left(\mu_{k+1}^{(i)}\right)^{T}\left(\tilde{G}_{k+1}^{(i)}\right)^{T} \tilde{G}_{k+1}^{(i)} C_{k+1}^{(i)} x_{k+1}^{(i)}\right] \\
& \left.+\mathbb{E}\left[-\varphi^{T}\left(C_{k+1}^{(i)} x_{k+1}^{(i)}\right)\left(\tilde{G}_{k+1}^{(i)}\right)^{T} \tilde{G}_{k+1}^{(i)} C_{k+1}^{(i)} x_{k+1}^{(i)}\right]\right\} \\
\leq & \left(1+\eta_{1}+\eta_{2}\right) \operatorname{tr}\left\{\mathbb{E}\left[\mu_{k+1}^{(i)}\left(\mu_{k+1}^{(i)}\right)^{T}\left(\tilde{G}_{k+1}^{(i)}\right)^{T} \tilde{G}_{k+1}^{(i)}\right]\right. \\
& +\left(1+\eta_{1}^{-1}+\eta_{3}\right) \operatorname{tr}\left\{\mathbb { E } \left[\varphi\left(C_{k+1}^{(i)} x_{k+1}^{(i)}\right)\right.\right. \\
& \left.\times \varphi^{T}\left(C_{k+1}^{(i)} x_{k+1}^{(i)}\right)\left(\tilde{G}_{k+1}^{(i)}\right)^{T} \tilde{G}_{k+1}^{(i)}\right]+\left(1+\eta_{2}^{-1}+\eta_{3}^{-1}\right) \\
& \times \operatorname{tr}\left\{\mathbb{E}\left[x_{k+1}^{(i)}\left(x_{k+1}^{(i)}\right)^{T}\left(C_{k+1}^{(i)}\right)^{T}\left(\tilde{G}_{k+1}^{(i)}\right)^{T} \tilde{G}_{k+1}^{(i)} C_{k+1}^{(i)}\right]\right\} \\
\leq & \left(\bar{k}^{2}+\bar{\beta} \bar{k}_{s}^{2}\right)\left[\left(1+\eta_{1}+\eta_{2}\right) m \bar{\phi}+\left(1+\eta_{1}^{-1}+\eta_{3}\right) \bar{\rho} m\right. \\
& \left.+\left(1+\eta_{2}^{-1}+\eta_{3}^{-1}\right) \bar{v}_{x} \bar{c}^{2}\right] \triangleq \bar{g}
\end{aligned}
$$

where

$\Lambda_{k+1}^{(i)} \triangleq\left(\Omega_{k+1}^{(i)}\right)^{T} \Omega_{k+1}^{(i)}+\beta_{k+1}^{(i)}\left(C_{k+1}^{(i)}\right)^{T}\left(G_{s, k+1}^{(i)}\right)^{T} G_{s, k+1}^{(i)} C_{k+1}^{(i)}$, $F_{k+1}^{(i)} \triangleq\left(G_{k+1}^{(i)}\right)^{T} G_{k+1}^{(i)}+\beta_{k+1}^{(i)}\left(G_{s, k+1}^{(i)}\right)^{T} G_{s, k+1}^{(i)}$.

In the sequel, an iterative matrix equation is constructed as follows:

$$
\Theta_{k+1}^{(i)}=\Omega_{k+1}^{(i)} A_{k}^{(i)} \Theta_{k}^{(i)}\left(A_{k}^{(i)}\right)^{T}\left(\Omega_{k+1}^{(i)}\right)^{T}+\Phi_{k+1}^{(i)}
$$

where $\Theta_{0}^{(i)} \triangleq B_{0}^{(i)} Q_{0}^{(i)}\left(B_{0}^{(i)}\right)^{T}+b I(b>0)$ and

$$
\begin{aligned}
& \Phi_{k+1}^{(i)} \\
\triangleq & G_{s, k+1}^{(i)} C_{k+1}^{(i)} A_{k}^{(i)} \Theta_{k}^{(i)}\left(A_{k}^{(i)}\right)^{T}\left(C_{k+1}^{(i)}\right)^{T}\left(G_{s, k+1}^{(i)}\right)^{T} \\
& +\sum_{j=1}^{L} M_{i} \bar{\zeta}_{k}^{(i j)} G_{s, k+1}^{(i)} C_{k+1}^{(i)} \Gamma \Theta_{k}^{(j)} \Gamma^{T}\left(C_{k+1}^{(i)}\right)^{T}\left(G_{s, k+1}^{(i)}\right)^{T} \\
& +\sum_{j=1}^{L} M_{i} \bar{\zeta}_{k}^{(i j)} \Omega_{k+1}^{(i)} \Gamma \Theta_{k}^{(j)} \Gamma^{T}\left(\Omega_{k+1}^{(i)}\right)^{T} \\
& +B_{k}^{(i)} Q_{k}^{(i)}\left(B_{k}^{(i)}\right)^{T}+b I .
\end{aligned}
$$

Now, we are in a position to prove the boundedness of $\Theta_{k+1}^{(i)}$. Let

$$
\tilde{\Theta}_{k} \triangleq \operatorname{diag}\left\{\Theta_{k}^{(1)}, \Theta_{k}^{(2)}, \cdots, \Theta_{k}^{(L)}\right\}
$$

then $\tilde{\Theta}_{k+1}$ satisfies

$$
\begin{aligned}
\tilde{\Theta}_{k+1}= & \Pi_{k+1} \tilde{A}_{k} \tilde{\Theta}_{k} \tilde{A}_{k}^{T} \Pi_{k+1}^{T} \\
& +\tilde{G}_{s, k+1} \tilde{C}_{k+1} \tilde{A}_{k} \tilde{\Theta}_{k} \tilde{A}_{k}^{T} \tilde{C}_{k+1}^{T} \tilde{G}_{s, k+1}^{T}
\end{aligned}
$$




$$
\begin{aligned}
& +\sum_{i=1}^{L} E_{i} \tilde{M}_{i} \Delta_{k}^{(i)} \Pi_{k+1}^{(i)} \tilde{\Gamma} \tilde{\Theta}_{k} \tilde{\Gamma}^{T}\left(\Pi_{k+1}^{(i)}\right)^{T} E_{i}^{T} \\
& +\sum_{i=1}^{L} E_{i} \tilde{M}_{i} \Delta_{k}^{(i)} \tilde{G}_{s, k+1}^{(i)} \tilde{C}_{k+1}^{(i)} \tilde{\Gamma} \tilde{\Theta}_{k} \tilde{\Gamma}^{T} \\
& \times\left(\tilde{C}_{k+1}^{(i)}\right)^{T}\left(\tilde{G}_{s, k+1}^{(i)}\right)^{T} E_{i}^{T}+\tilde{B}_{k} \tilde{Q}_{k} \tilde{B}_{k}^{T}+\mathscr{E} .
\end{aligned}
$$

Taking the norm for the both sides of (57) leads to

$$
\begin{aligned}
& \left\|\tilde{\Theta}_{k+1}\right\| \\
\leq & {\left[\left\|\Pi_{k+1}\right\|^{2}\left\|\tilde{A}_{k}\right\|^{2}+\left\|\tilde{G}_{s, k+1}\right\|^{2}\left\|\tilde{C}_{k+1}\right\|^{2}\left\|\tilde{A}_{k}\right\|^{2}\right.} \\
& +\sum_{i=1}^{L}\left\|E_{i}\right\|^{2}\left\|\tilde{M}_{i}\right\|\left\|\Delta_{k}^{(i)}\right\|\left(\left\|\Pi_{k+1}^{(i)}\right\|^{2}\|\tilde{\Gamma}\|^{2}+\left\|\tilde{G}_{s, k+1}^{(i)}\right\|^{2}\right. \\
& \left.\left.\times\left\|\tilde{C}_{k+1}^{(i)}\right\|^{2}\|\tilde{\Gamma}\|^{2}\right)\right]\left\|\tilde{\Theta}_{k}\right\|+\left\|\tilde{B}_{k}\right\|^{2}\left\|\tilde{Q}_{k}\right\|+b \\
\leq & \delta_{1}\left\|\tilde{\Theta}_{k}\right\|+\delta_{2}
\end{aligned}
$$

where

$$
\begin{aligned}
& \delta_{1} \triangleq\left(\bar{m}^{2}+\bar{k}_{s}^{2} \bar{c}^{2}\right)\left(\bar{a}^{2}+L^{2} \bar{t} \bar{\zeta} \bar{r}^{2}\right), \\
& \delta_{2} \triangleq \bar{b} \bar{q}+b .
\end{aligned}
$$

It follows from (56) that

$$
\Theta_{k+1}^{(i)}=\bar{e}_{i} \tilde{\Theta}_{k+1} \bar{e}_{i}^{T}
$$

and, subsequently,

$$
\left\|\Theta_{k+1}^{(i)}\right\| \leq\left\|\tilde{\Theta}_{k+1}\right\| \leq \delta_{1}\left\|\tilde{\Theta}_{k}\right\|+\delta_{2} .
$$

If $\delta_{1}<1$, the following inequality can be obtained

$$
\left\|\Theta_{k+1}^{(i)}\right\| \leq\left\|\tilde{\Theta}_{0}\right\|+\frac{\delta_{2}}{1-\delta_{1}} .
$$

On the other hand, (54)-(55) tells

$$
\Theta_{k+1}^{(i)} \geq b I .
$$

In light of (61) and (62), there exist scalars $\underline{\Theta}$ and $\bar{\Theta}$ such that

$$
\underline{\Theta} I \leq \Theta_{k+1}^{(i)} \leq \bar{\Theta} I
$$

holds for all $k \geq 0$.

Now, we are in a position to focus on the proof of the boundedness of the filtering errors. Let us define

$$
V_{k}\left(e_{k}\right)=\sum_{i=1}^{L}\left(\varepsilon_{k}^{(i)}\right)^{T}\left(\Theta_{k}^{(i)}\right)^{-1} \varepsilon_{k}^{(i)}
$$

where

$$
e_{k} \triangleq \operatorname{col}\left\{\varepsilon_{k}^{(1)}, \cdots, \varepsilon_{k}^{(L)}\right\} .
$$

According to (63), it is clear that

$$
\bar{\Theta}^{-1}\left\|e_{k}\right\|^{2} \leq \mathbb{E}\left[V_{k}\left(e_{k}\right)\right] \leq \underline{\Theta}^{-1}\left\|e_{k}\right\|^{2} .
$$

For positive scalars $\eta_{4}$ and $\eta_{5}$, according to (11) and (49), we obtain the following inequality:

$$
\begin{aligned}
& \mathbb{E}\left[V_{k+1}\left(e_{k+1}\right) \mid e_{k}\right]-\left(1+\eta_{4}+\eta_{5}\right) V_{k}\left(e_{k}\right) \\
\leq & \left(1+\eta_{4}+\eta_{5}\right) \sum_{i=1}^{L} \mathbb{E}\left\{( \varepsilon _ { k } ^ { ( i ) } ) ^ { T } \left[\left(A_{k}^{(i)}\right)^{T}\left(\Omega_{k+1}^{(i)}\right)^{T}\left(\Theta_{k+1}^{(i)}\right)^{-1}\right.\right.
\end{aligned}
$$

$$
\begin{aligned}
& \left.\times \Omega_{k+1}^{(i)} A_{k}^{(i)}-\left(\Theta_{k}^{(i)}\right)^{-1}\right]\left(\varepsilon_{k}^{(i)}\right)^{T} \\
& +\beta_{k+1}^{(i)}\left(\varepsilon_{k}^{(i)}\right)^{T}\left(A_{k}^{(i)}\right)^{T}\left(C_{k+1}^{(i)}\right)^{T}\left(G_{s, k+1}^{(i)}\right)^{T} \\
& \left.\times\left(\Theta_{k+1}^{(i)}\right)^{-1} G_{s, k+1}^{(i)} C_{k+1}^{(i)} A_{k}^{(i)} \varepsilon_{k}^{(i)}\right\}+\left(1+\eta_{4}^{-1}+\eta_{6}\right) \\
& \times \sum_{i=1}^{L} \sum_{j=1}^{L} c^{2} M_{i} \bar{\zeta}_{k}^{(i j)} \mathbb{E}\left[\left(\varepsilon_{k}^{(j)}\right)^{T} \Gamma^{T} M_{k+1}^{(i)} \Gamma \varepsilon_{k}^{(j)}\right] \\
& +\left(1+\eta_{7}\right) \sum_{i=1}^{L} \mathbb{E}\left[\left(\gamma_{k+1}^{(i)}\right)^{T}\left(\Theta_{k+1}^{(i)}\right)^{-1} \gamma_{k+1}^{(i)}\right] \\
& +\left(1+\eta_{5}^{-1}+\eta_{6}^{-1}+\eta_{7}^{-1}\right) \sum_{i=1}^{L} \mathbb{E}\left[\left(g_{k+1}^{(i)}\right)^{T}\left(\Theta_{k+1}^{(i)}\right)^{-1} g_{k+1}^{(i)}\right]
\end{aligned}
$$

where

$$
\begin{aligned}
M_{k+1}^{(i)}= & \left(\Omega_{k+1}^{(i)}\right)^{T}\left(\Theta_{k+1}^{(i)}\right)^{-1} \Omega_{k+1}^{(i)}+\beta_{k+1}^{(i)}\left(C_{k+1}^{(i)}\right)^{T} \\
& \times\left(G_{s, k+1}^{(i)}\right)^{T}\left(\Theta_{k+1}^{(i)}\right)^{-1} G_{s, k+1}^{(i)} C_{k+1}^{(i)} .
\end{aligned}
$$

Utilizing the matrix inversion lemma, we have

$$
\begin{aligned}
& \left(A_{k}^{(i)}\right)^{T}\left(\Omega_{k+1}^{(i)}\right)^{T}\left(\Theta_{k+1}^{(i)}\right)^{-1} \Omega_{k+1}^{(i)} A_{k}^{(i)}-\left(\Theta_{k}^{(i)}\right)^{-1} \\
= & \left(A_{k}^{(i)}\right)^{T}\left(\Omega_{k+1}^{(i)}\right)^{T}\left[\Omega_{k+1}^{(i)} A_{k}^{(i)} \Theta_{k}^{(i)}\left(A_{k}^{(i)}\right)^{T}\left(\Omega_{k+1}^{(i)}\right)^{T}\right. \\
& \left.+\Phi_{k+1}^{(i)}\right]^{-1} \Omega_{k+1}^{(i)} A_{k}^{(i)}-\left(\Theta_{k}^{(i)}\right)^{-1} \\
= & -\left[\Theta_{k}^{(i)}+\Theta_{k}^{(i)}\left(A_{k}^{(i)}\right)^{T}\left(\Omega_{k+1}^{(i)}\right)^{T}\left(\Phi_{k+1}^{(i)}\right)^{-1} \Omega_{k+1}^{(i)} A_{k}^{(i)} \Theta_{k}^{(i)}\right]^{-1} \\
= & -\left[I+\left(A_{k}^{(i)}\right)^{T}\left(\Omega_{k+1}^{(i)}\right)^{T}\left(\Phi_{k+1}^{(i)}\right)^{-1} \Omega_{k+1}^{(i)} A_{k}^{(i)} \Theta_{k}^{(i)}\right]^{-1}\left(\Theta_{k}^{(i)}\right)^{-1} \\
\leq & -\left(1+\frac{\bar{a}^{2} \bar{m}^{2} \bar{\Theta}}{\underline{q} \underline{b}}\right)^{-1}\left(\Theta_{k}^{(i)}\right)^{-1} \triangleq \bar{f}\left(\Theta_{k}^{(i)}\right)^{-1} .
\end{aligned}
$$

On the other hand, it follows from the expression of $\Theta_{k+1}^{(i)}$ in (54) that

$$
\begin{aligned}
& \Theta_{k+1}^{(i)}>G_{s, k+1}^{(i)} C_{k+1}^{(i)} A_{k}^{(i)} \Theta_{k}^{(i)}\left(A_{k}^{(i)}\right)^{T}\left(C_{k+1}^{(i)}\right)^{T}\left(G_{s, k+1}^{(i)}\right)^{T}, \\
& \Theta_{k+1}^{(i)} \geq M_{i} \bar{\zeta}_{k}^{(i j)} \Omega_{k+1}^{(i)} \Gamma \Theta_{k}^{(j)} \Gamma^{T}\left(\Omega_{k+1}^{(i)}\right)^{T}, \\
& \Theta_{k+1}^{(i)} \geq M_{i} \bar{\zeta}_{k}^{(i j)} G_{s, k+1}^{(i)} C_{k+1}^{(i)} \Gamma \Theta_{k}^{(j)} \Gamma^{T}\left(C_{k+1}^{(i)}\right)^{T}\left(G_{s, k+1}^{(i)}\right)^{T} .
\end{aligned}
$$

Then, the second and third terms on the right-hand side of (67) are rewritten as

$$
\begin{aligned}
& \beta_{k+1}^{(i)}\left(\varepsilon_{k}^{(i)}\right)^{T}\left(A_{k}^{(i)}\right)^{T}\left(C_{k+1}^{(i)}\right)^{T}\left(G_{s, k+1}^{(i)}\right)^{T}\left(\Theta_{k+1}^{(i)}\right)^{-1} \\
& \times G_{s, k+1}^{(i)} C_{k+1}^{(i)} A_{k}^{(i)} \varepsilon_{k}^{(i)} \\
\leq & \bar{\beta}\left(\varepsilon_{k}^{(i)}\right)^{T}\left(\Theta_{k}^{(i)}\right)^{-1} \varepsilon_{k}^{(i)}
\end{aligned}
$$

and

$$
\begin{aligned}
& \sum_{i=1}^{L} \sum_{j=1}^{L} c^{2} M_{i} \bar{\zeta}_{k}^{(i j)} \mathbb{E}\left[\left(\varepsilon_{k}^{(j)}\right)^{T} \Gamma^{T} M_{k+1}^{(i)} \Gamma \varepsilon_{k}^{(j)}\right] \\
\leq & c^{2} \sum_{i=1}^{L} \sum_{j=1}^{L} \mathbb{E}\left[\left(1+\beta_{k+1}^{(i)}\right)\left(\varepsilon_{k}^{(j)}\right)^{T}\left(\Theta_{k}^{(j)}\right)^{-1} \varepsilon_{k}^{(j)}\right] \\
\leq & c^{2} L(1+\bar{\beta}) \sum_{j=1}^{L} \mathbb{E}\left[\left(\varepsilon_{k}^{(j)}\right)^{T}\left(\Theta_{k}^{(j)}\right)^{-1} \varepsilon_{k}^{(j)}\right] .
\end{aligned}
$$

Substituting (69)-(72) into (67) yields

$$
\mathbb{E}\left[V_{k+1}\left(e_{k+1}\right) \mid e_{k}\right]-\left(1+\eta_{4}+\eta_{5}\right) V_{k}\left(e_{k}\right)
$$




$$
\begin{aligned}
\leq & \left(1+\eta_{4}+\eta_{5}\right)(\bar{f}+\bar{\beta}) \sum_{i=1}^{L} \mathbb{E}\left\{\left(\varepsilon_{k}^{(i)}\right)^{T}\left(\Theta_{k}^{(i)}\right)^{-1}\left(\varepsilon_{k}^{(i)}\right)^{T}\right\} \\
& +\left(1+\eta_{4}^{-1}+\eta_{6}\right) c^{2}(1+\bar{\beta}) L \\
& \times \sum_{i=1}^{L} \mathbb{E}\left\{\left(\varepsilon_{k}^{(i)}\right)^{T}\left(\Theta_{k}^{(i)}\right)^{-1}\left(\varepsilon_{k}^{(i)}\right)^{T}\right\} \\
& +\left(1+\eta_{7}\right) L \bar{\gamma} \underline{\Theta}^{-1}+\left(1+\eta_{5}^{-1}+\eta_{6}^{-1}+\eta_{7}^{-1}\right) L \bar{g} \underline{\Theta}^{-1} \\
= & \varrho V_{k}\left(e_{k}\right)+\lambda
\end{aligned}
$$

where

$$
\begin{aligned}
& \varrho \triangleq\left(1+\eta_{4}+\eta_{5}\right)(\bar{f}+\bar{\beta})+\left(1+\eta_{4}^{-1}+\eta_{6}\right) c^{2}(1+\bar{\beta}) L, \\
& \lambda \triangleq\left(1+\eta_{7}\right) L \bar{\gamma} \underline{\Theta}^{-1}+\left(1+\eta_{5}^{-1}+\eta_{6}^{-1}+\eta_{7}^{-1}\right) L \bar{g} \underline{\Theta}^{-1} .
\end{aligned}
$$

Then, it follows from (71) that

$$
\mathbb{E}\left[V_{k+1}\left(e_{k+1}\right) \mid e_{k}\right] \leq \nu V_{k}\left(e_{k}\right)+\lambda
$$

with $\nu$ being defined in (48). In addition, we can immediately obtain

$$
\begin{aligned}
\mathbb{E}\left[\left\|e_{k+1}\right\|^{2}\right] & \leq \frac{\bar{\Theta}}{\underline{\Theta}} \mathbb{E}\left[\left\|e_{0}\right\|^{2}\right] \nu^{k+1}+\lambda \bar{\Theta} \sum_{i=1}^{k} \nu^{i} \\
& \leq \frac{\bar{\Theta}}{\underline{\Theta}} \mathbb{E}\left[\left\|e_{0}\right\|^{2}\right] \nu^{k+1}+\frac{\lambda \bar{\Theta}}{1-\nu}
\end{aligned}
$$

which indicates that $\varepsilon_{k}^{(i)}$ is exponentially bounded in the sense of mean-square if $\nu<1$.

Remark 5: A sufficient condition has been provided in Theorem 3 to guarantee the mean-square boundedness of $e_{k}$. It is worthwhile to note that $\Phi_{k+1}^{(i)}$ is not only dependent on $\Theta_{k}^{(i)}$ but also on $\Theta_{k}^{(j)}(j=1,2, \cdots, L)$, and this brings additional difficulties to discover the relationship between $\left\|\Theta_{k+1}^{(i)}\right\|$ and $\left\|\Theta_{k}^{(i)}\right\|$. To deal with such a challenge, the term $\tilde{\Theta}_{k+1}$ has been introduced in (54) and the recurrence relation of $\tilde{\Theta}_{k+1}$ has been determined as in (57). Then, it is not difficult to verify the boundedness of $\Theta_{k+1}^{(i)}$.

\section{ILlustrative Examples}

Example 1: Consider a $\mathrm{CN}$ consisting of 3 coupled nodes with known matrices:

$$
\begin{aligned}
& A_{k}^{(1)}=\left[\begin{array}{cc}
0.95 & 1.24 \\
-0.5 & 0.92 \sin (5 k)
\end{array}\right], A_{k}^{(2)}=\left[\begin{array}{cc}
-0.65 & -0.3 \\
0.2+0.5 \sin (3 k) & -0.3
\end{array}\right], \\
& A_{k}^{(3)}=\left[\begin{array}{cc}
0.38 & -0.7 \\
0.5 & 0.1
\end{array}\right], B_{k}^{(1)}=\left[\begin{array}{c}
0.1 \\
-0.6
\end{array}\right], B_{k}^{(2)}=\left[\begin{array}{l}
-0.1 \\
-0.9
\end{array}\right], \\
& B_{k}^{(3)}=\left[\begin{array}{c}
0.1 \\
-0.6
\end{array}\right], C_{k}^{(1)}=\left[\begin{array}{c}
0.5 \\
1
\end{array}\right]^{T}, C_{k}^{(2)}=\left[\begin{array}{c}
2.4 \\
4.5
\end{array}\right]^{T}, C_{k}^{(3)}=\left[\begin{array}{l}
1 \\
1
\end{array}\right], \\
& \Gamma=\left[\begin{array}{cc}
0.1 & 0 \\
0 & 0.1
\end{array}\right], G_{s, k}^{(1)}=\left[\begin{array}{c}
0.2 \\
0.1
\end{array}\right], G_{s, k}^{(2)}=\left[\begin{array}{c}
0.05 \\
0.2
\end{array}\right], G_{s, k}^{(3)}=\left[\begin{array}{l}
0.3 \\
0.2
\end{array}\right] .
\end{aligned}
$$

In this simulation, we set $c=8 \times 10^{-3}, e_{1}=e_{2}=$ $e_{6}=e_{7}=e_{8}=e_{9}=0.2, e_{3}=e_{4}=e_{5}=0.5$. The initial conditions are $x_{1}^{(0)}=\hat{x}_{1 \mid 0}^{(1)}=[0.5,1]^{T}, x_{2}^{(0)}=\hat{x}_{1 \mid 0}^{(2)}=$ $[-1,0.25]^{T}, x_{3}^{(0)}=\hat{x}_{1 \mid 0}^{(3)}=[-0.5,-0.75]^{T}, \Sigma_{0 \mid 0}^{(1)}=0.01 I_{2}$, $\Sigma_{0 \mid 0}^{(2)}=0.02 I_{2}$ and $\Sigma_{0 \mid 0}^{(3)}=0.01 I_{2}$. Meanwhile, we set $Q_{k}^{(i)}=0.01, R_{k}^{(i)}=0.01, \bar{\zeta}_{k}^{(i i)}=0.95,(i=1,2,3)$, $\bar{\zeta}_{k}^{(12)}=0.85, \bar{\zeta}_{k}^{(13)}=0.65, \bar{\zeta}_{k}^{(21)}=0.75, \bar{\zeta}_{k}^{(23)}=0.55$, $\bar{\zeta}_{k}^{(31)}=0.85, \bar{\zeta}_{k}^{(32)}=0.75, \beta_{k}^{(i)}=1(i=1,2,3)$. The dynamic event-triggered parameters are chosen as $h_{i}=0.1$, $\sigma_{i}=0.01$ and $\theta_{i}=0.1(i=1,2,3)$. In addition, the saturation level parameters are $\alpha_{i}=1, \iota_{i}=1(i=1,2,3), \rho_{1}=0.5$, $\rho_{2}=0.8$ and $\rho_{3}=0.9$.

Figs. 1-2 display trajectories of actual states and their estimates. It is clear that the true trajectories are well tracked by the designed filter. Based on Theorems 1-2, the upper bounds are obtained, and the curves of $\log$ (the actual error covariance) and their bounds are given in Figs. 3-4, from which we spot that curves of $\log ($ MSE) stay below that of upper bounds. In addition, Figs. 5-6 plot the curves of the actual filtering error covariances with hope to better display the changing trend of their filtering errors, which shows that the filtering errors are small and tend to be stable.

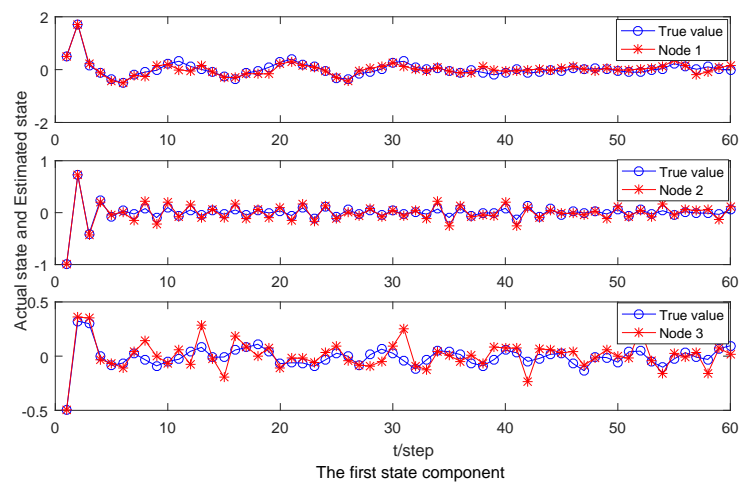

Fig. 1. The curves of $x_{1, k}^{(i)}$ and $\hat{x}_{1, k}^{(i)}$ on node $i(i=1,2,3)$.

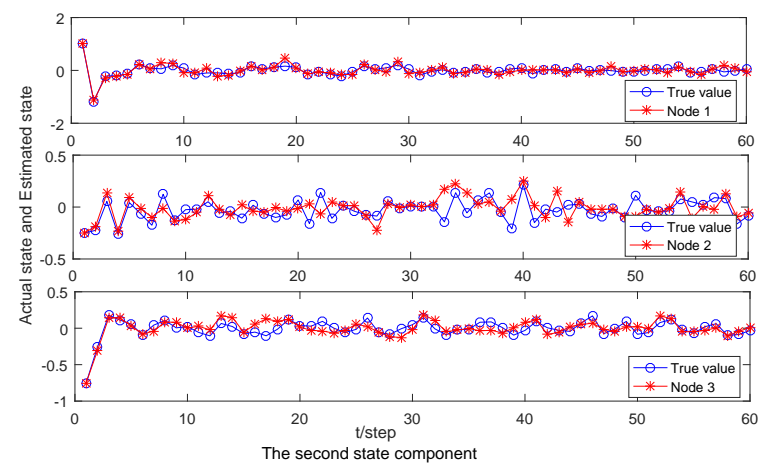

Fig. 2. The curves of $x_{2, k}^{(i)}$ and $\hat{x}_{2, k}^{(i)}$ on node $i(i=1,2,3)$.

Example 2: The presented dynamic event-triggered nonfragile filtering approach is applied to a network of interconnected flexible link robot systems [8], [35], where the system state is composed of the angular position of the motor shaft $\theta_{m, k}^{(i)}$, the velocity of the motor shaft $\vartheta_{m, k}^{(i)}$, the angular position of the link $\theta_{l, k}^{(i)}$ and the velocity of the link $\vartheta_{l, k}^{(i)}$ $(i=1,2, \cdots, 5)$. Discretizating the original system under the sampling period $T=1$, the following system parameters 


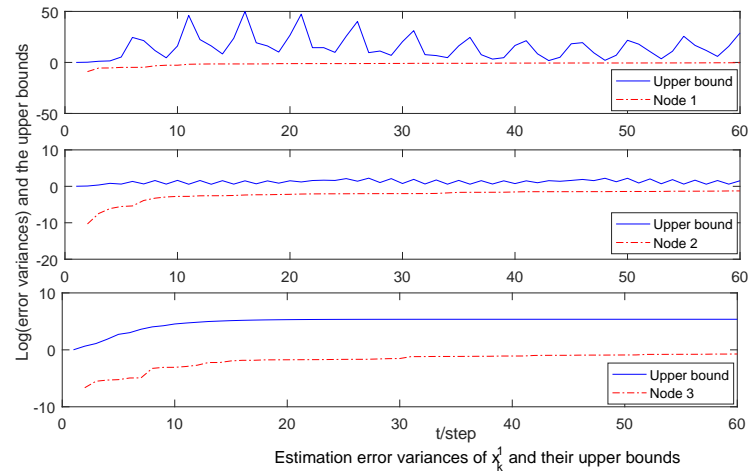

Fig. 3. $\log ($ the actual error covariance) and their corresponding upper bounds.

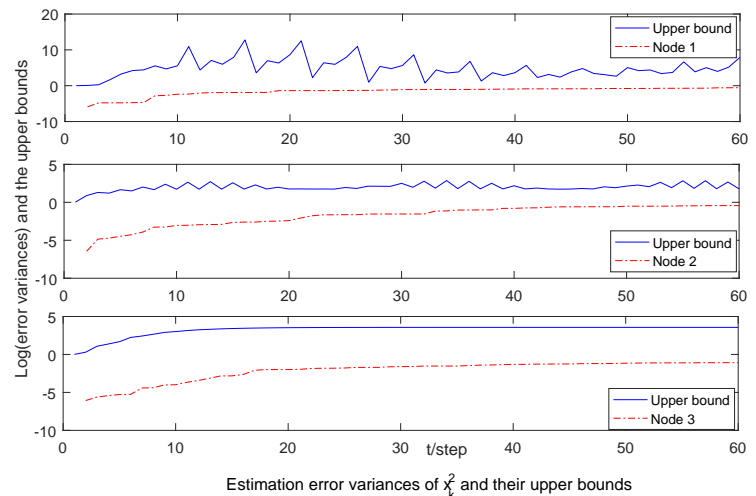

Fig. 4. $\log$ (the actual error covariance) and their corresponding upper bounds.

are obtained:

$$
\begin{aligned}
A_{i}(k) & =\left[\begin{array}{cccc}
-0.0727 & 0.0002 & 0.2095 & -0.0240 \\
-0.4842 & -0.0751 & 1.2996 & 0.2095 \\
-0.0362 & -0.0098 & 0.2351 & 0.0562 \\
1.5873 & 0.0852 & -3.4969 & 0.2351
\end{array}\right], \\
C_{i}(k) & =\left[\begin{array}{llll}
1 & 0 & 0 & 0 \\
0 & 1 & 0 & 0
\end{array}\right],
\end{aligned}
$$

Other matrices are given as:

$B_{k}^{(1)}=\left[\begin{array}{llll}0.1 & -0.6 & 0.2 & 0.3\end{array}\right], B_{k}^{(2)}=\left[\begin{array}{llll}-0.1 & -0.9 & 0.4 & 0.1\end{array}\right]$, $B_{k}^{(3)}=\left[\begin{array}{llll}0.1 & -0.6 & 0.3 & 0.5\end{array}\right], B_{k}^{(4)}=\left[\begin{array}{llll}0.3 & 0.1 & 0 & 0.05\end{array}\right]$, $B_{k}^{(5)}=\left[\begin{array}{llll}0.9 & -0.1 & 0.7 & 0.6\end{array}\right], G_{s, k}^{(i)}=\left[\begin{array}{llll}0.9 & 0.5 & 0.3 & 0.5 \\ 0.1 & 0.1 & 0.2 & 0.3\end{array}\right]^{T}$.

The initial values are $x_{1}^{(0)}=\hat{x}_{1 \mid 0}^{(1)}=[0.5,1,0,0]^{T}$, $x_{2}^{(0)}=\hat{x}_{1 \mid 0}^{(2)}=[-1,0.25,0,0]^{T}, x_{3}^{(0)}=\hat{x}_{1 \mid 0}^{(3)}=$ $[-0.5,-0.75,0,0]^{T}, x_{4}^{(0)}=\hat{x}_{1 \mid 0}^{(4)}=[0.1,0,0.5,0]^{T}, x_{5}^{(0)}=$ $\hat{x}_{1 \mid 0}^{(5)}=[-0.5,0.25,0,1]^{T}$, and $\Sigma_{0 \mid 0}^{(i)}=0.1 I_{4},(i=1, \cdots, 5)$. In addition, we set $c=8 \times 10^{-3}, e_{i}=0.2, Q_{k}^{(i)}=0.01$, $R_{k}^{(i)}=0.01, \bar{\zeta}_{k}^{(i i)}=0.95, \bar{\zeta}_{k}^{(12)}=0.85, \bar{\zeta}_{k}^{(13)}=0.65, \bar{\zeta}_{k}^{(14)}=$ $0.5, \bar{\zeta}_{k}^{(15)}=0.85, \bar{\zeta}_{k}^{(21)}=0.75, \bar{\zeta}_{k}^{(23)}=0.55, \bar{\zeta}_{k}^{(24)}=0.55$, $\bar{\zeta}_{k}^{(23)}=0.65, \bar{\zeta}_{k}^{(31)}=0.85, \bar{\zeta}_{k}^{(32)}=0.75, \bar{\zeta}_{k}^{(34)}=0.75$,

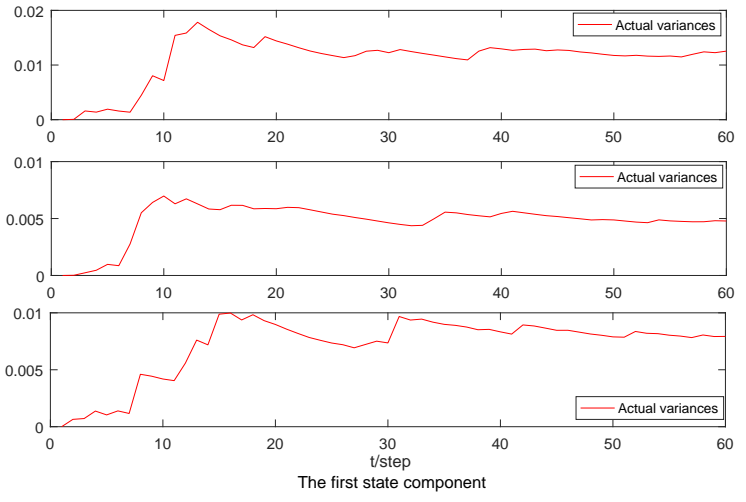

Fig. 5. The actual filtering error variance for the first state component.

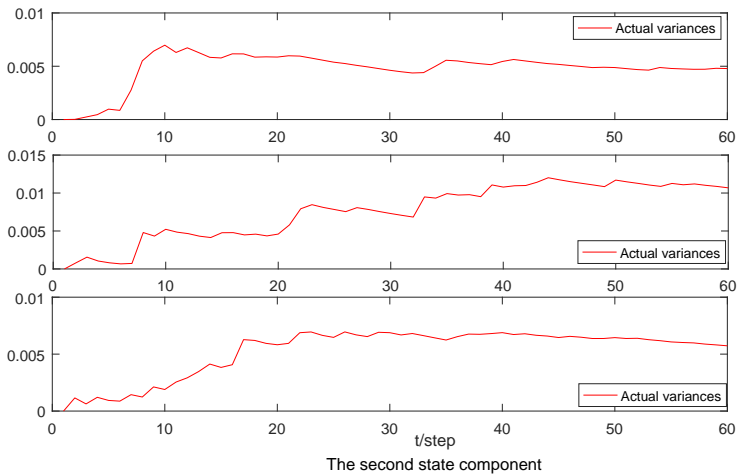

Fig. 6. The actual estimation error variance for the second state component. $\bar{\zeta}_{(j 5)}^{(35)}=0.95, \bar{\zeta}_{k}^{(41)}=0.75, \bar{\zeta}_{k}^{(42)}=0.95, \bar{\zeta}_{k}^{(43)}=0.55$,
$\bar{\zeta}_{k}^{(45)}=0.55, \bar{\zeta}_{k}^{(51)}=0.85, \bar{\zeta}_{k}^{(52)}=0.75, \bar{\zeta}_{k}^{(53)}=0.95$, $\bar{\zeta}_{k}^{(54)}=0.65$. The dynamic event-triggered parameters are chosen as $h_{i}=0.1, \sigma_{1}=0.2, \sigma_{2}=0.05, \sigma_{3}=0.1, \sigma_{4}=0.15$ and $\sigma_{5}=0.01$. and $\theta_{i}=10$. The saturation level parameters are $\alpha_{i}=0.1, \iota_{i}=0.1, \rho_{1}=0.5, \rho_{2}=0.8, \rho_{3}=0.9, \rho_{4}=0.6$ and $\rho_{5}=0.4$.

Figs. 7-10 demonstrate trajectories of actual states and their estimates, which show the true trajectories are well tracked by the proposed filter. Moreover, Fig. 11 plots the dynamic event-triggered time. It is observed that the transmitted data become less as the threshold increases.

\section{CONCLUSIONS}

In this paper, we have solved the non-fragile filtering problem for CNs with sensor saturations and switching topologies. The DECP has been adopted to adaptively tune triggering thresholds, thereby saving communication costs. Upper bounds on error covariances have been derived and minimized by properly choosing gain parameters. Sufficient conditions have been obtained to guarantee mean-square boundedness of error dynamics. Finally, simulations have been given to prove the usefulness of our developed filtering algorithm. One of our future research topics would be the extension of the presented filtering method to systems with more complicated networkinduced phenomena [3], [12]. 


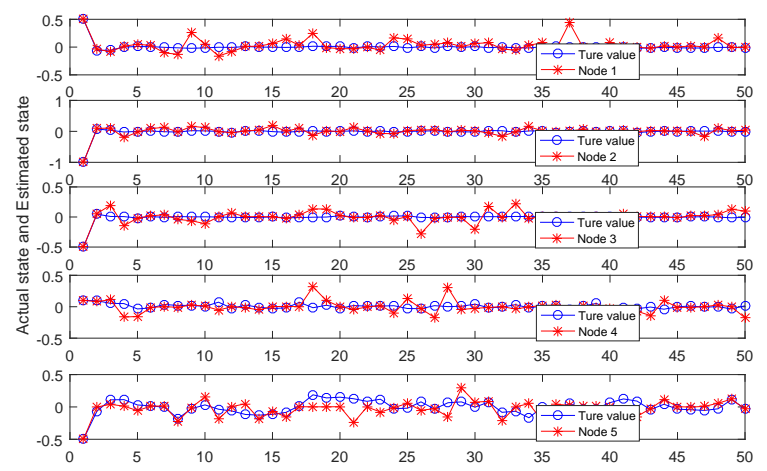

Fig. 7. The curves of $x_{1, k}^{(i)}$ and $\hat{x}_{1, k}^{(i)}$ on node $i(i=1, \cdots, 5)$.

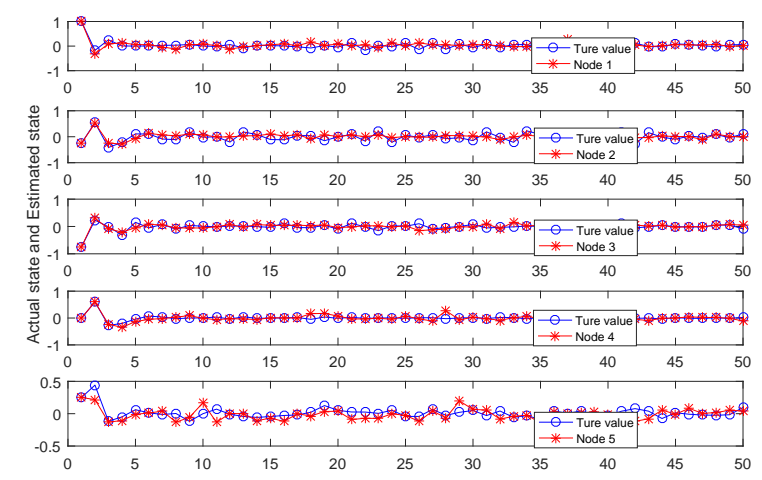

Fig. 8. The curves of $x_{2, k}^{(i)}$ and $\hat{x}_{2, k}^{(i)}$ on node $i(i=1, \cdots, 5)$.

\section{REFERENCES}

[1] M. V. Basin, P. C. R. Ramirez and F. Guerra-Avellaneda, Continuous fixed-time controller design for mechatronic systems with incomplete measurements, IEEE-ASME Transactions on Mechatronics, vol. 23, no. 1, pp. 57-67, 2018.

[2] R. Caballero-Águila, A. Hermoso-Carazo and J. Linares-Pérez, Distributed fusion filters from uncertain measured outputs in sensor networks with random packet losses, Information Fusion, vol. 34, pp. 7079, 2017.

[3] Y. Chen, Z. Wang, L. Wang and W. Sheng, Mixed $H_{2} / H_{\infty}$ state estimation for discrete-time switched complex networks with random coupling strengths through redundant channels, IEEE Transactions on Neural Networks and Learning Systems, vol 31, no. 10, pp. 4130-4142, 2020.

[4] D. Ding, Z. Wang and Q.-L. Han, Neural-network-based consensus control for multiagent systems with input constraints: The event-triggered case, IEEE Transactions on Cybernetics, vol. 50, no. 8, pp. 3719-3730, 2020.

[5] D. Ding, Z. Wang and Q.-L. Han, A scalable algorithm for eventtriggered state estimation with unknown parameters and switching topologies over sensor networks, IEEE Transactions on Cybernetics, vol. 50, no. 9, pp. 4087-4097, 2020.

[6] H. Dong, N. Hou, Z. Wang and W. Ren, Variance-constrained state estimation for complex networks with randomly varying topologies, IEEE Transactions on Neural Networks and Learning Systems,vol. 29, no. 7 , pp. $2757-2768,2018$.

[7] R. Duan and J. Li, Finite-time distributed $H_{\infty}$ filtering for TakagiSugeno fuzzy system with uncertain probability sensor saturation under switching network topology: Non-PDC approach, Applied Mathematics and Computation, vol. 371, article no. 124961, 2020.

[8] C. Edwards and P. P. Menon, On distributed pinning observers for a network of dynamical systems, IEEE Transactions on Automatic Control, vol. 61, no. 12, pp. 4081-4087, 2016.

[9] X. Ge, Q.-L. Han and Z. Wang, A dynamic event-triggered transmission scheme for distributed set-membership estimation over wireless sensor

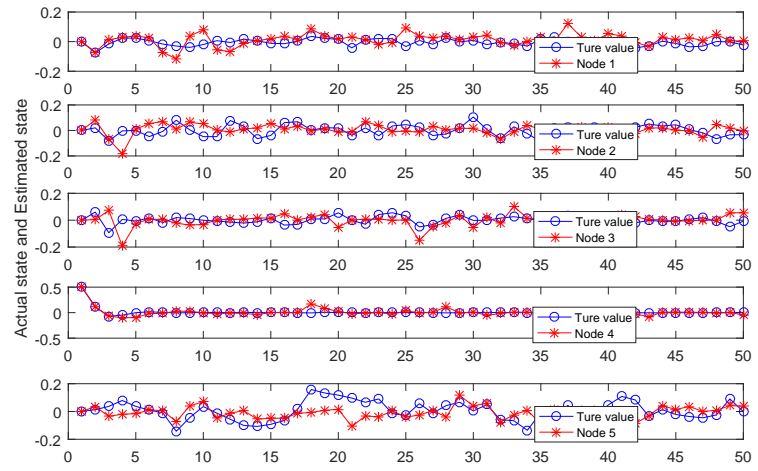

Fig. 9. The curves of $x_{3, k}^{(i)}$ and $\hat{x}_{3, k}^{(i)}$ on node $i(i=1, \cdots, 5)$.

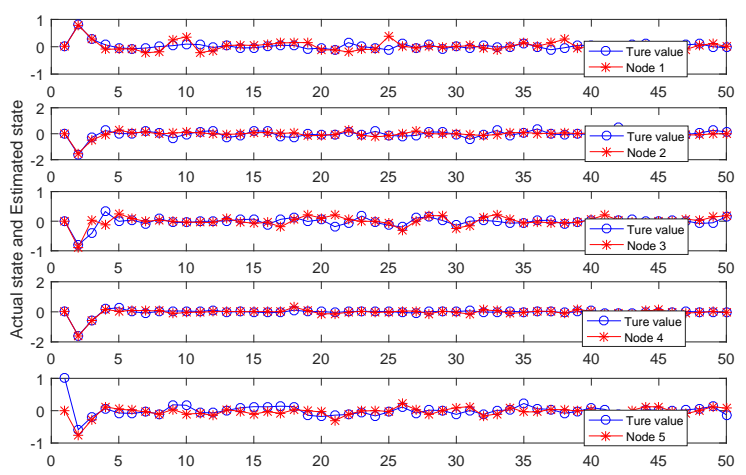

Fig. 10. The curves of $x_{4, k}^{(i)}$ and $\hat{x}_{4, k}^{(i)}$ on node $i(i=1, \cdots, 5)$.

networks, IEEE Transactions on Cybernetics, vol. 49, no. 1, pp. 171183,2019

[10] A. Girard, Dynamic triggering mechanisms for event-triggered control, IEEE Transactions on Automatic Control, vol. 60, no. 7, pp. 1992-1997, 2015.

[11] X. He, W. Xue, X. Zhang and H. Fang, Distributed filtering for uncertain systems under switching sensor networks and quantized communications, Automatica, vol. 114, article no. 108842, 2020.

[12] N. Hou, Z. Wang, D. W. C. Ho and H. Dong, Robust partial-nodes-based state estimation for complex networks under deception attacks, IEEE Transactions on Cybernetics, vol. 50, no. 6, pp. 2793-2802, 2020.

[13] J. Hu, Z. Wang, G.-P. Liu, C. Jia and J. Williams, Event-triggered recursive state estimation for dynamical networks under randomly switching topologies and multiple missing measurements, Automatica, vol. 115, article no. 108908, 2020.

[14] J. Hu, Z. Wang, G.-P. Liu and H. Zhang, Variance-constrained recursive state estimation for time-varying complex networks with quantized measurements and uncertain inner coupling, IEEE Transactions on Neural Networks and Learning Systems, vol. 31, no. 6, pp. 1955-1967, 2020.

[15] J. Hu, Z. Wang, S. Liu and H. Gao, A variance-constrained approach to recursive state estimation for time-varying complex networks with missing measurements, Automatica, vol. 64, pp. 155-162, 2016.

[16] C. Huang, B. Shen, H. Chen and H. Shu, A dynamically eventtriggered approach to recursive filtering with censored measurements and parameter uncertainties, Journal of the Franklin Institute, vol. 356, no. 15, pp. 8870-8889, 2019.

[17] R. Konrad, G. Stefan, Y. Engin and R. Unbehauen, Stochastic stability of the discrete-time extended Kalman filter, IEEE Transactions on Automatic Control, vol. 44, no. 4, pp. 714-728, 1999.

[18] Q. Li, Z. Wang, W. Sheng, F. E. Alsaadi and F. E. Alsaadi, Dynamic event-triggered mechanism for $H_{\infty}$ non-fragile state estimation of complex networks under randomly occurring sensor saturations, Information Sciences, vol. 509, pp. 304-316, 2020.

[19] Q. Li, Z. Wang, N. Li and W. Sheng, A dynamic event-triggered 


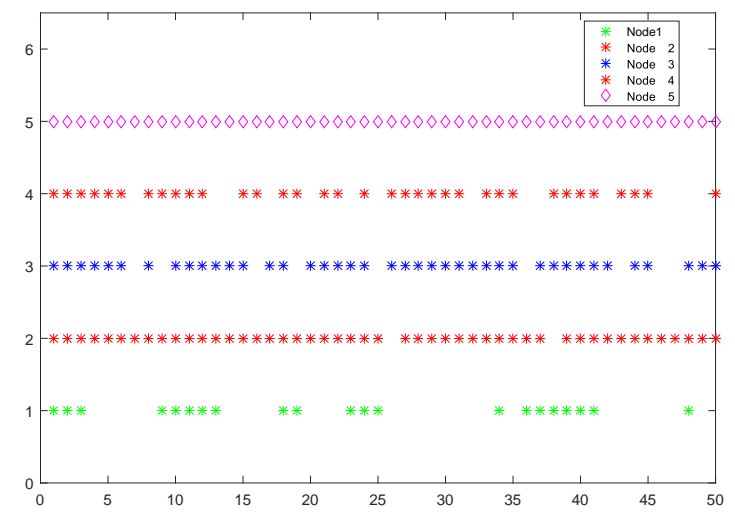

Fig. 11. The event-triggered triggering instants.

approach to recursive filtering for complex networks with switching topologies subject to random sensor failures, IEEE Transactions on Neural Networks and Learning Systems, vol. 31. no. 10, pp. 4381-4388, 2020.

[20] W. Li, Y. Jia and J. Du, State estimation for stochastic complex networks with switching topology, IEEE Transactions on Automatic Control, vol. 62, no. 12, pp. 6377-6384, 2017.

[21] Z. Lian, P. Shi and Y. Sun, Design on nonfragile fuzzy filter for nonlinear time-delay systems, International Journal of Adaptive Control and Signal Processing, vol. 34, no. 2, pp. 248-265, 2020.

[22] D. Liu, Y. Liu and E. F. Alsaadi, Recursive state estimation based-on the outputs of partial nodes for discrete-time stochastic complex networks with switched topology, Journal of the Franklin Institute, vol. 355, no. 11, pp. 4686-4707, 2018.

[23] H. Liu, Z. Wang, W. Fei and J. Li, Resilient $H_{\infty}$ state estimation for discrete-time stochastic delayed memristive neural Networks: A dynamic event-triggered mechanism, IEEE Transactions on Cybernetics, in press, DOI: 10.1109/TCYB.2020.3021556.

[24] J. Liu, Y. Gu, L. Zha, Y. Liu and J. Cao, Event-triggered $H_{\infty}$ load frequency control for multiarea power systems under hybrid cyber attacks, IEEE Transactions on Systems Man Cybernetics: Systems, vol. 49, no. 8, pp. 1665-1678, 2019.

[25] Q. Liu and Z. Wang, Moving-horizon estimation for linear dynamic networks with binary encoding schemes, IEEE Transactions on Automatic Control, in press, DOI: 10.1109/TAC.2020.2996579.

[26] Q. Liu, Z. Wang, X. He, G. Ghinea and F. E. Alsaadi, A resilient approach to distributed filter design for time-varying systems under stochastic nonlinearities and sensor degradation, IEEE Transactions on Signal Processing, vol. 65, no. 5, pp. 1300-1309, 2017.

[27] X. Liu, D. W. C. Ho and C. Xie, Prespecified-Time cluster synchronization of complex networks via a smooth control approach, IEEE Transactions on Cybernetics, vol. 50, no. 4, pp. 1771-1775, 2020.

[28] Y. Liu, B. Shen and H. Shu, Finite-time resilient $H_{\infty}$ state estimation for discrete-time delayed neural networks under dynamic event-triggered mechanism, Neural Networks, vol. 121, pp. 356-365, 2020.

[29] Y. Liu, Z. Wang, L. Ma and F. E. Alsaadi, A partial-nodes-based information fusion approach to state estimation for discrete-time delayed stochastic complex networks, Information Fusion, vol. 49, pp. 240-248, 2019.

[30] Y. Liu, Z. Wang, Y. Yuan and W. Liu, Event-triggered partial-nodesbased state estimation for delayed complex networks with bounded distributed delays, IEEE Transactions on Systems, Man, and Cybernetics: Systems, vol. 49, no. 6, pp. 1088-1098, 2019.

[31] Y. Luo, Z. Wang, G. Wei and F. E. Alsaadi, Non-fragile $\ell_{2}-\ell_{\infty}$ fault estimation for Markovian jump 2-D systems with specified power bounds, IEEE Transactions on Systems, Man, and Cybernetics: Systems, vol. 50, no. 5, pp. 1964-1975, 2020.

[32] L. Ma, Z. Wang, J. Hu and Q.-L. Han, Probability-guaranteed envelopeconstrained filtering for nonlinear systems subject to measurement outliers, IEEE Transactions on Automatic Control, in press, DOI: 10.1109/TAC.2020.3016767.

[33] J. Mao, D. Ding, Y. Song, Y. Liu and F. E. Alsaadi, Event-based recursive filtering for time-delayed stochastic nonlinear systems with missing measurements, Signal Processing, vol. 134, pp. 158-165, 2017.

[34] B. Qu, Z. Wang and B. Shen, Fusion estimation for a class of multi-rate power systems with randomly occurring SCADA measurement delays, Automatica, vol. 125, art. no. 109408, 2021.

[35] S. Raghavan and J. K. Hedrick, Observer design for a class of nonlinear systems, International Journal of Control, vol. 59, no. 2, pp. 515-528, 1994.

[36] B. Shen, Z. Wang, D. Wang and Q. Li, State-saturated recursive filter design for stochastic time-varying nonlinear complex networks under deception attacks, IEEE Transactions on Neural Networks and Learning Systems, vol. 31, no. 10, pp. 3788-3800, 2020.

[37] J. Song, D. Ding, H. Liu and X. Wang, Non-fragile distributed state estimation over sensor networks subject to DoS attacks: The almost sure stability, International Journal of Systems Science, vol. 51, no. 6, pp. 1119-1132, 2020 .

[38] X. Wan, Z. Wang, M. Wu and X. Liu, $H_{\infty}$ state estimation for discretetime nonlinear singularly perturbed complex networks under the RoundRobin protocol, IEEE Transactions on Neural Networks and Learning Systems, vol. 30, no. 2, pp. 415-426, 2019.

[39] F. Wang and J. Liang, Constrained $H_{\infty}$ estimation for time-varying networks with hybrid incomplete information, International Journal of Robust and Nonlinear Control, vol. 28, no. 2, pp. 699-715, 2018.

[40] J.-L. Wang, Z. Qin, H.-N. Wu and T. Huang, Finite-Time synchronization and $H_{\infty}$ synchronization of multiweighted complex networks with adaptive state couplings, IEEE Transactions on Cybernetics, vol. 50, no. 2, pp. 600-612, 2020.

[41] L. Wang, Z. Wang, B. Shen and G. Wei, Recursive filtering with measurement fading: A multiple description coding scheme, IEEE Transactions on Automatic Control, in press, DOI: 10.1109/TAC.2020.3034196.

[42] M. Wang, Z. Wang, Y. Chen and W. Sheng, Adaptive neural eventtriggered control for discrete-time strict-feedback nonlinear systems, IEEE Transactions on Cybernetics, vol. 50, no. 7, pp. 2946-2958, 2020.

[43] X. Wang and G. Chen, Complex networks: small-world, scale-free, and Beyond, IEEE Circuits and Systems Magazine, vol. 3, no. 1, pp. 6-20, 2003.

[44] D. Watts and S. Strogatz, Collective dynamics of small-world networks, Nature, vol. 393, pp. 440-442, 1998.

[45] J. Xia, G. Chen, J. H. Park, H. Shen and G. Zhuang, Dissipativitybased sampled-data control for fuzzy switched markovian jump systems, IEEE Transactions on Fuzzy Systesms, in press, DOI: 10.1109/TFUZZ.2020.2970856.

[46] N. Yang, D. Chen, D. Ji and Z. Wu, Resilient state estimation for nonlinear complex networks with time-delay under stochastic communication protocol, Neurocomputing,vol. 346, pp. 38-47, 2019.

[47] J. Zhang, X. He and D. Zhou, Robust filtering and intermittent fault detection for time-verying systems with stochastic parameter uncertainty and sensor saturation, International Federation of Automatic Control, vol. 51, no. 24, pp. 1324-1329, 2018.

[48] W. Zhang, X. Yang and C. Li, Fixed-Time stochastic synchronization of complex networks via continuous control, IEEE Transactions on Cybernetics, vol. 49, no. 8, pp. 3099-3104, 2019.

[49] X.-M. Zhang, Q.-L. Han, X. Ge, D. Ding, L. Ding, D. Yue and C. Peng, Networked control systems: A survey of trends and techniques, IEEECAA Journal of Automatica Sinica, vol. 7, no. 1, pp. 1-17, 2020.

[50] D. Zhao, Z. Wang, G. Wei and X. Liu, Nonfragile $H_{\infty}$ state estimation for recurrent neural networks with time-varying delays: On proportionalintegral observer design, IEEE Transactions on Neural Networks and Learning Systems, in press, DOI: 10.1109/TNNLS.2020.3015376.

[51] Z. Zhao, Z. Wang, L. Zou and J. Guo, Set-Membership filtering for timevarying complex networks with uniform quantisations over randomly delayed redundant channels, International Journal of Systems Science, in press, DOI: 10.1080/00207721.2020.1814898.

[52] L. Zou, Z. Wang, Q.-L. Han and D. H. Zhou, Moving horizon estimation of networked nonlinear systems with random access protocol, IEEE Transactions on Systems, Man, and Cybernetics: Systems, in press, DOI 10.1109/TSMC.2019.2918002.

[53] L. Zou, Z. Wang and D. H. Zhou, Moving horizon estimation with non-uniform sampling under component-based dynamic event-triggered transmission, Automatica, vol. 120, art. no. 109154, 2020.

[54] Z. Zuo, P. Xie and Y. Wang, Output-based dynamic event-triggering control for sensor saturated systems with external disturbance, Applied Mathematics and Computation, vol. 374, article no. 125043, 2020. 


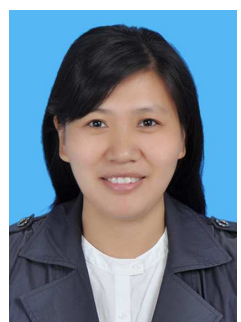

Shaoying Wang received her B.Sc. degree in mathematics in 2004 from Linyi University, Linyi, China, and the M.Sc. degree in applied mathematics in 2006 from Wuhan University, Wuhan, China and the $\mathrm{Ph} . \mathrm{D}$. degree in control theory and control engineering in 2016 from Huazhong University of Science and Technology, Wuhan, China. She is currently an associate professor in the College of Science, Binzhou University, Shandong, China, and is also a postdoctoral student in the College of Electrical and Information Engineering, Northeast Petroleum University. Her research interests include networked control systems and state estimation.

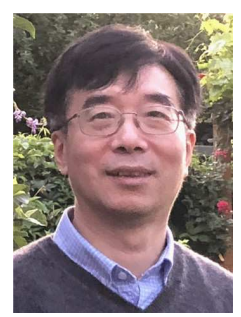

Zidong Wang (SM'03-F'14) was born in Jiangsu, China, in 1966. He received the B.Sc. degree in mathematics in 1986 from Suzhou University, Suzhou, China, and the M.Sc. degree in applied mathematics in 1990 and the Ph.D. degree in electrical engineering in 1994, both from Nanjing University of Science and Technology, Nanjing, China.

$\mathrm{He}$ is currently Professor of Dynamical Systems and Computing in the Department of Computer Science, Brunel University London, U.K. From 1990 to 2002, he held teaching and research appointments in universities in China, Germany and the UK. Prof. Wang's research interests include dynamical systems, signal processing, bioinformatics, control theory and applications. He has published more than 600 papers in international journals. He is a holder of the Alexander von Humboldt Research Fellowship of Germany, the JSPS Research Fellowship of Japan, William Mong Visiting Research Fellowship of Hong Kong.

Prof. Wang serves (or has served) as the Editor-in-Chief for International Journal of Systems Science, the Editor-in-Chief for Neurocomputing, and an Associate Editor for 12 international journals including IEEE Transactions on Automatic Control, IEEE Transactions on Control Systems Technology, IEEE Transactions on Neural Networks, IEEE Transactions on Signal Processing, and IEEE Transactions on Systems, Man, and Cybernetics-Part C. He is a Member of the Academia Europaea, a Fellow of the IEEE, a Fellow of the Royal Statistical Society and a member of program committee for many international conferences.

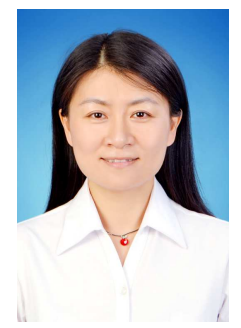

Hongli Dong received the Ph.D. degree in control science and engineering from the Harbin Institute of Technology, Harbin, China, in 2012.

From 2009 to 2010, she was a Research Assistant with the Department of Applied Mathematics, City University of Hong Kong, Hong Kong. From 2010 to 2011, she was a Research Assistant with the Department of Mechanical Engineering, The University of Hong Kong, Hong Kong. From 2011 to 2012, she was a Visiting Scholar with the Department of Information Systems and Computing, Brunel University London, London, U.K. From 2012 to 2014, she was an Alexander von Humboldt Research Fellow with the University of Duisburg-Essen, Duisburg, Germany. She is currently a Professor with the Artificial Intelligence Energy Research Institute, Northeast Petroleum University, Daqing, China. She is also the Director of the Heilongjiang Provincial Key Laboratory of Networking and Intelligent Control, Daqing. Her current research interests include robust control and networked control systems.

Dr. Dong is a very active reviewer for many international journals.

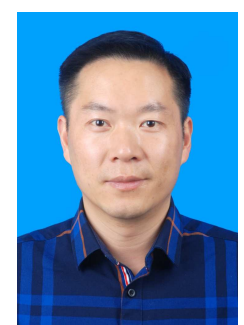

Yun Chen received the B.E. degree in thermal engineering in 1999 from Central South University of Technology (Central South University), Changsha, China, and the M.E. degree in engineering thermal physics in 2002 and Ph.D. degree in control science and engineering in 2008 , both from Zhejiang University, Hangzhou, China.

From August 2009 to August 2010, he was a visiting fellow with the School of Computing, Engineering and Mathematics, University of Western Sydney, Australia. From December 2016 to December 2017 he was an academic visitor with the Department of Mathematics, Brunel University London, UK. In 2002, he joined Hangzhou Dianzi University, China, where he is currently a Professor. His research interests include stochastic and hybrid systems, robust control and filtering. 